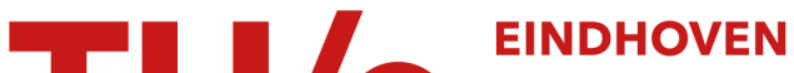 UNIVERSITY OF TECHNOLOGY
}

\section{Generating-function approach for double freeform lens design}

\section{Citation for published version (APA):}

Romijn, L. B., ten Thije Boonkkamp, J. H. M., Anthonissen, M. J. H., \& IJzerman, W. L. (2021). Generatingfunction approach for double freeform lens design. Journal of the Optical Society of America A, Optics, Image Science and Vision, 38(3), 356-368. https://doi.org/10.1364/JOSAA.411883

\section{Document license:}

TAVERNE

DOI:

10.1364/JOSAA.411883

Document status and date:

Published: 01/03/2021

\section{Document Version:}

Publisher's PDF, also known as Version of Record (includes final page, issue and volume numbers)

\section{Please check the document version of this publication:}

- A submitted manuscript is the version of the article upon submission and before peer-review. There can be important differences between the submitted version and the official published version of record. People interested in the research are advised to contact the author for the final version of the publication, or visit the $\mathrm{DOI}$ to the publisher's website.

- The final author version and the galley proof are versions of the publication after peer review.

- The final published version features the final layout of the paper including the volume, issue and page numbers.

Link to publication

\section{General rights}

Copyright and moral rights for the publications made accessible in the public portal are retained by the authors and/or other copyright owners and it is a condition of accessing publications that users recognise and abide by the legal requirements associated with these rights.

- Users may download and print one copy of any publication from the public portal for the purpose of private study or research.

- You may not further distribute the material or use it for any profit-making activity or commercial gain

- You may freely distribute the URL identifying the publication in the public portal.

If the publication is distributed under the terms of Article $25 \mathrm{fa}$ of the Dutch Copyright Act, indicated by the "Taverne" license above, please follow below link for the End User Agreement:

www.tue.nl/taverne

\section{Take down policy}

If you believe that this document breaches copyright please contact us at:

openaccess@tue.nl

providing details and we will investigate your claim. 


\title{
Generating-function approach for double freeform lens design
}

\author{
L. B. RomiJn, ${ }^{1, *}$ M. J. H. Anthonissen, ${ }^{1}$ J. H. M. Ten ThiJe BoonkKamp,${ }^{1}$ And \\ W. L. IJZERMAN ${ }^{1,2}$ \\ ${ }^{1}$ CASA, Department of Mathematics and Computer Science, Eindhoven University of Technology, P.O. Box 513, 5600 MB \\ Eindhoven, The Netherlands \\ ${ }^{2}$ Signify Research, High Tech Campus 7, 5656 AE Eindhoven, The Netherlands \\ *Corresponding author: I.b.romijn@tue.nl
}

Received 8 October 2020; revised 18 December 2020; accepted 22 December 2020; posted 12 January 2021 (Doc. ID 411883 ); published 11 February 2021

\begin{abstract}
Many LED lighting applications involve the design of multiple optical surfaces. A prime example is a single lens with two refractive surfaces. In this paper, we consider an LED light source approximated as a point and a far-field target intensity. Using Hamilton's characteristic functions, the design problem is converted into two generalized Monge-Ampère equations by deriving a generating function for each optical surface. The generating function is a generalization of the cost function in optimal transport theory. The generalized Monge-Ampère equations are solved using an iterative least-squares algorithm. To compute the first optical surface, we choose an intermediate far-field target intensity. By choosing different intermediate target intensities based on the source and target intensity, we develop a "knob" to distribute the refractive power over both surfaces of the lens. We apply the algorithm on two example problems and show it is capable of producing complicated target distributions. () 2021 Optical Society of America
\end{abstract}

https://doi.org/10.1364/JOSAA.411883

\section{INTRODUCTION}

The design of multiple freeform surfaces is gaining traction in illumination optics design, with the aim to produce more advanced and compact luminaires. In this paper, we use a point light source approximation in the freeform lens design for LED applications and a far-field target. We compute the shape of two freeform surfaces of a lens using a Monge-Ampère-based approach.

For many optical systems, we can derive an equation of the form

$$
u_{2}(\boldsymbol{y})-u_{1}(\boldsymbol{x})=c(\boldsymbol{x}, \boldsymbol{y}) .
$$

The source coordinates $\boldsymbol{x} \in \mathcal{X}$ are related to the position or direction of the rays incident on an optical surface, and the target coordinates $\boldsymbol{y} \in \mathcal{Y}$ are related to the position or direction of the rays reflected or refracted off the same surface. In Eq. (1), $u_{1}(\boldsymbol{x})$ is a parametrization of a surface in terms of the source coordinates $\boldsymbol{x} \in \mathcal{X}, u_{2}(\boldsymbol{y})$ is a geometrical variable related to the target coordinates $\boldsymbol{y} \in \mathcal{Y}$, and $c(\boldsymbol{x}, \boldsymbol{y})$ is an optimal transport cost function. However, not all optical systems belong to this category. Prime examples of optical systems that cannot be put in this optimal transport framework are systems with a near-field target. Also, the equation describing the location of the second freeform surface of the lens presented in this paper cannot be put in the optimal transport framework. Instead, we formulate a generating function $G$ of the form

$$
u_{1}(\boldsymbol{x})=G\left(\boldsymbol{x}, \boldsymbol{y}, u_{2}(\boldsymbol{y})\right),
$$

one for each surface of the lens. The generating functions can be derived using Hamilton's characteristic functions of optical path length (OPL). Combining an optimal transport equation Eq. (1) or a generating function Eq. (2) with energy conservation leads to a nonlinear second-order partial differential equation, also called a generalized Monge-Ampère equation. Generalized Monge-Ampère equations associated with generating functions are also called generated Jacobian equations, and the generating functions are generalizations of cost functions in optimal transport theory [1]. Trudinger [2] introduced the framework of generated Jacobian equations and drew examples from geometrical optics that cannot be formulated as optimal transport problems.

Generated Jacobian equations can be written in terms of the underlying generating functions and are solved numerically using a least-squares algorithm. Originally, this method was developed for the standard Monge-Ampère equation [3], considering the design of a single reflector or lens for a parallel incoming source beam and far-field target, and corresponding to a quadratic cost function in optimal transport theory. The numerical procedure was extended to non-quadratic cost functions in [4] (parallel-to-parallel double freeform lens) and 
[5,6] (point-to-far-field single reflector/lens). The cost-function approach was extended to a generating-function approach in [1] and tested on a near-field problem. Currently, the algorithm is applicable to any optical system that can be described by a smooth generating function. The least-squares procedure iteratively optimizes the optical map and surface using a formulation involving the unique inverse $H$ of the generating function $G$, such that $u_{1}(\boldsymbol{x})=G\left(\boldsymbol{x}, \boldsymbol{y}, H\left(\boldsymbol{x}, \boldsymbol{y}, u_{1}(\boldsymbol{x})\right)\right.$. In fact, the function $H$ is equal to Hamilton's characteristic function.

In this paper, we use the least-squares procedure to design double freeform lenses for a point source and far-field target, where the incident wavefront is spherical and the outgoing wavefront has a general shape. We derive two generating functions, one for each optical surface. First, we run the least-squares procedure to compute the first optical surface and a mapping from the source domain to an intermediate target domain. Subsequently, we run the least-squares procedure again to compute the second optical surface and a mapping from the source domain to the final target domain. We have an extra degree of freedom in the design by choosing an intermediate target intensity to compute the shape of the first optical surface. We will present an example problem where we progressively translate and scale the intermediate target distribution from the source to the final target distribution, showing the effect of distributing the refractive power over two optical surfaces. Motivated by practical examples, the aim of this paper is to introduce the generating-function approach to compute a double freeform lens and demonstrate the flexibility of our numerical algorithm to compute multiple solutions that differ in design. In future work, we aim to apply our algorithm in an engineering setting, and analyze whether the resulting designs satisfy compactness requirements and are suitable for injection molding.

This paper is structured as follows. In the next section, we provide a brief overview of methods available to compute double freeform surfaces. In Section 2, we present the derivation of the generating functions and corresponding generated Jacobian equations. The generalized least-squares method is described in Section 3. In Section 4, we test the performance of the algorithm. Finally, we make some concluding remarks in Section 5 .

\section{A. Literature Review}

The literature on inverse methods to compute freeform optical surfaces is extensive and can roughly be divided into three categories: (1) methods that directly solve the generalized Monge-Ampère equation of the optical system [7-10], (2) optimization strategies for the corresponding Monge-Kantorovich mass transportation problem [4-6,11-22], and (3) methods that indirectly compute the surface by using ray mapping techniques [23-28]. For an in-depth overview, we refer to [6]. We mention a few numerical methods that are designed to compute double freeform surfaces. Such optical systems can be further categorized according to the shape of the source and target wavefronts, i.e., (A) collimated and/or spherical, and (B) at least one wavefront of general shape. We consider only light sources with zero-étendue, which is conserved throughout the system [29]. Methods in category (A) considering collimated and point source and target distributions are applied to laser beam shaping problems. The input beam may be collimated or spherical, and the output beam is also collimated or converging to a point. The aim is to construct two freeform surfaces to control the intensity distribution or phase profile of the beam. For collimated and/or point sources and targets, if the shape of one of the two freeform surfaces is known, the other one directly follows using the Theorem of Malus and Dupin (principle of equal OPL) ([30], p. 130), or by showing that one of Hamilton's characteristic functions is constant.

Using the categories of inverse methods from the previous paragraph, we mention a few laser beam shaping methods: (1.A) Zhang et al. [31] derive the generalized Monge-Ampère equation and solve the corresponding nonlinear boundary value problem using Newton's method. Yadav et al. [4] present a formulation of the generalized Monge-Ampère equation by first deriving the non-quadratic cost function of the optical system in optimal transport theory. The freeform surfaces are computed with a least-squares method using this cost function as input. (2.A) Oliker et al. [32] use the Monge-Kantorovich formulation of the problem and compute a unique solution using the supporting quadric method, which involves a pixelation of the target domain and an iterative adjustment of the parameters of tangent quadrics to optical surfaces. Moiseev et al. [33] design a collimator with two freeform optical surfaces and introduce an angular deflection parameter to control the refractive contributions of each optical surface. The surfaces are calculated using Oliker's method of supporting paraboloids and the principle of equal OPL. It was found that the most efficient angular deflection occurs when the required angular deflection is divided equally among the two surfaces, such that the total Fresnel losses are minimized. Doskolovich et al. [13,14] reduce the optimal transport problem in its discrete version to a linear assignment problem (LAP) for the mapping, by constructing an equal-flux grid in the source and target domains. The LAP is solved using methods such as the Hungarian algorithm and auction algorithm. B-splines are used to compute the surface from the mapping. (3.A) Feng et al. [34,35] first compute a ray mapping by using an adaptive mesh method to numerically solve the standard Monge-Ampère equation, i.e., $\operatorname{det}\left(D^{2} u\right)=f(\boldsymbol{x}, u, \nabla u)$, with $D^{2} u$ the Hessian matrix of $u$ and $f$ a positive function. A ray mapping $\boldsymbol{m}=\nabla u$ is computed. Subsequently, the two freeform optical surfaces are constructed simultaneously. The first surface is constructed point by point using the ray mapping and Snell's law, and the second surface follows by equaling the OPLs between the input and output wavefronts. The construction of the first freeform surface is analogous to Euler's method for solving ordinary differential equations (ODEs) and the simultaneous multiple surfaces (SMS) method in three dimensions to connect the wavefronts [36]. The initial mapping and surfaces are improved in an iterative procedure to find an integrable ray mapping and to improve the initial ray mapping, since it is calculated using the standard Monge-Ampère equation and not the generalized Monge-Ampère equation. Ray mapping techniques work well under the paraxial and thin lens approximations.

Numerical methods for double freeform surfaces with a source and/or target wavefront which is non-collimated or not a singular point are scarce. To the best of our knowledge, we are the first to present a method in category (1.B) and to introduce 
an extra degree of freedom in the system design. As far as we know, there are no methods in category (2.B). (3.B) Examples of ray mapping techniques are: Wei et al. [37] consider source distributions with wavefronts of general shape (i.e., non-collimated and non-spherical source intensities). The authors use the leastsquares algorithm in [3] to compute an initial mapping and a point-by-point procedure to compute an initial approximation to the first freeform surface. The second freeform surface is constructed according to the principle of equal OPL. The initial mapping and surfaces are improved in an iterative procedure to find integrable normal vectors to the surfaces, i.e., a surface can be constructed with unit tangent vectors perpendicular to the unit normal vectors derived from the mapping. Bruneton et al. [38] consider a point source distribution, and using an initial ray mapping, the two freeform surfaces are constructed with a least-squares optimization algorithm, but the details have been omitted. Similar to our approach, the authors distribute the refraction of the rays over two surfaces. However, Bruneton et al. [38] use an angular redirection of the rays, similar to Moiseev et al. [33], while optimizing the difference between the light flux that arrives on the target within triangular tubes of rays and the desired target distribution. In this paper, we use an intensity-based approach: we use an intermediate target intensity to compute the first surface, and subsequently the second surface with the final far-field target intensity. Last, we mention Gimenez et al. [36] who present an extension to the two-dimensional SMS method first described in [39]. The method builds upon the edge-ray principle [40], and, unlike the previously described methods, two input and two output light distributions are required for construction of the surfaces.

\section{MATHEMATICAL FORMULATION}

In this section, we derive the generating functions for the two freeform surfaces, which we will combine with energy conservation to derive the generalized Monge-Amperre equations.

Figure 1 schematically illustrates a point source, a lens with refractive index $n$, and a far-field target. A beam of light emanates from the point source located at $\mathcal{O}$ of the Cartesian coordinate system with $(x, y, z) \in \mathbb{R}$. The point source emits rays of light radially outward in the direction $\hat{\boldsymbol{s}}=\hat{\boldsymbol{e}}_{r}$, where $\hat{\boldsymbol{e}}_{r}$ is the radial basis vector in the spherical coordinate system. Unit vectors are denoted by hats.

The first surface of the lens is described by $\mathcal{L}_{1}: \boldsymbol{r}_{1}(\phi, \theta)=$ $u(\phi, \theta) \hat{\boldsymbol{e}}_{r}$, where $u(\phi, \theta)>0$ is the radial parameter that describes the location of the surface, $0 \leq \phi \leq \pi$ is the zenith, and $0 \leq \theta<2 \pi$ is the azimuth in the spherical coordinate system. The surface $\mathcal{L}_{1}$ refracts the ray $\hat{\boldsymbol{s}}$ in direction $\hat{\boldsymbol{\imath}}$.

The second surface of the lens is described by $\mathcal{L}_{2}: \boldsymbol{r}_{2}(\phi, \theta)=$ $u(\phi, \theta) \hat{\boldsymbol{e}}_{r}+v(\phi, \theta) \hat{\boldsymbol{\iota}}$, where $v(\phi, \theta)>0$ is the parameter that describes the location of the surface relative to the first surface and is equal to the distance $d\left(P_{1}, P_{2}\right)$ between the point $P_{1}\left(u(\phi, \theta) \hat{\boldsymbol{e}}_{r}\right)$ on the first surface and a point $P_{2}$; see Fig. 1 . The surface $\mathcal{L}_{2}$ refracts the ray $\hat{\imath}$ in direction $\hat{\boldsymbol{t}}$.

The intensity of the source is given by $f(\phi, \theta)[\mathrm{lm} / \mathrm{sr}]$. To compute the first surface, we consider an intermediate target intensity in the far field $h\left(\psi_{1}, \chi_{1}\right)[\mathrm{lm} / \mathrm{sr}]$, where $\left(\psi_{1}, \chi_{1}\right)$ represents a different set of spherical coordinates, with zenith

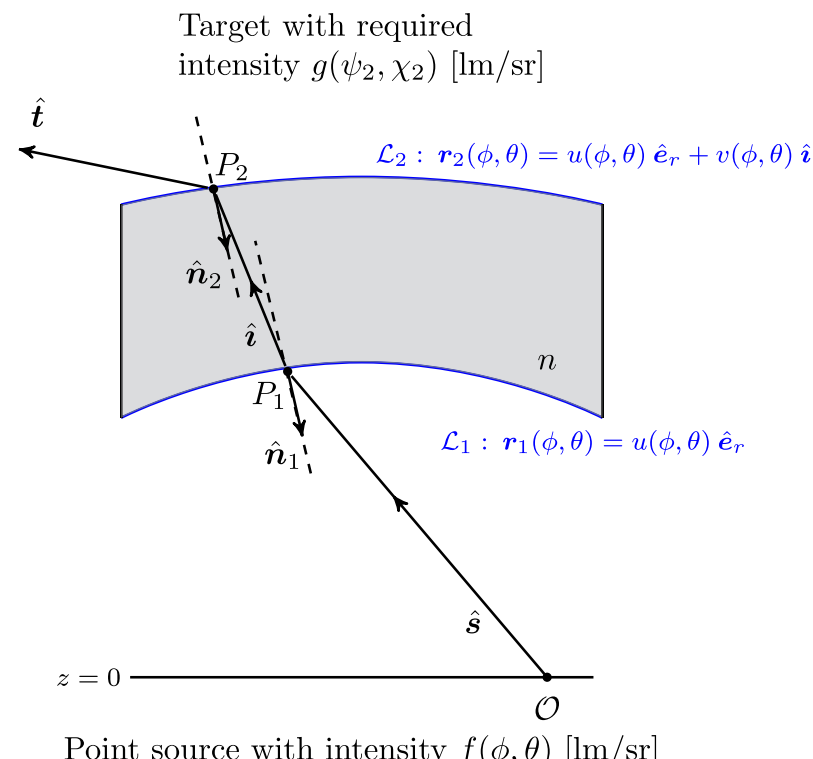

Fig. 1. Double freeform lens converting the intensity $f(\phi, \theta)$ of a point source into a far-field target intensity $g\left(\psi_{2}, \chi_{2}\right)$.

$0 \leq \psi_{1} \leq \pi$ and azimuth $0 \leq \chi_{1}<2 \pi$. Analogously, for the second surface the final target intensity in the far field is denoted by $g\left(\psi_{2}, \chi_{2}\right)[\mathrm{lm} / \mathrm{sr}]$, where $\left(\psi_{2}, \chi_{2}\right)$ represents yet another set of spherical coordinates, with zenith $0 \leq \psi_{2} \leq \pi$ and azimuth $0 \leq \chi_{2}<2 \pi$. The origin of the coordinate system describing the intermediate and final targets is the lens approximated as a point in space (i.e., all directions $\hat{\boldsymbol{\imath}}$ and $\hat{\boldsymbol{t}}$ emanate from the same point). This approximation is called the far-field approximation.

We transform the coordinates of the light rays from spherical to stereographic. This is convenient since the vectors $\hat{\boldsymbol{s}}=\left(s_{1}, s_{2}, s_{3}\right)^{\mathrm{T}}, \hat{\boldsymbol{\imath}}=\left(\iota_{1}, \iota_{2}, \iota_{3}\right)^{\mathrm{T}}$, and $\hat{\boldsymbol{t}}=\left(t_{1}, t_{2}, t_{3}\right)^{\mathrm{T}}$ are defined on the unit sphere $S^{2}$. Hence, $|\hat{\boldsymbol{s}}|=|\hat{\boldsymbol{\imath}}|=|\hat{\boldsymbol{t}}|=1$. We define

$$
\begin{aligned}
\boldsymbol{x}(\hat{\boldsymbol{s}}) & =\left(\begin{array}{l}
x_{1} \\
x_{2}
\end{array}\right) \\
& =\frac{1}{1+s_{3}}\left(\begin{array}{l}
s_{1} \\
s_{2}
\end{array}\right) \\
& =\frac{1}{1+\cos (\phi)}\left(\begin{array}{c}
\sin (\phi) \cos (\theta) \\
\sin (\phi) \sin (\theta)
\end{array}\right), \\
\boldsymbol{y}_{1}(\hat{\boldsymbol{\imath}}) & =\left(\begin{array}{l}
y_{11} \\
y_{12}
\end{array}\right)=\frac{1}{1+\iota_{3}}\left(\begin{array}{l}
\iota_{1} \\
\iota_{2}
\end{array}\right) \\
& =\frac{1}{1+\cos \left(\psi_{1}\right)}\left(\begin{array}{l}
\sin \left(\psi_{1}\right) \cos \left(\chi_{1}\right) \\
\sin \left(\psi_{1}\right) \sin \left(\chi_{1}\right)
\end{array}\right), \\
\boldsymbol{y}_{2}(\hat{\boldsymbol{t}}) & =\left(\begin{array}{l}
y_{21} \\
y_{22}
\end{array}\right)=\frac{1}{1+t_{3}}\left(\begin{array}{l}
t_{1} \\
t_{2}
\end{array}\right) \\
& =\frac{1}{1+\cos \left(\psi_{2}\right)}\left(\begin{array}{l}
\sin \left(\psi_{2}\right) \cos \left(\chi_{2}\right) \\
\sin \left(\psi_{2}\right) \sin \left(\chi_{2}\right)
\end{array}\right),
\end{aligned}
$$

with corresponding inverse projections 


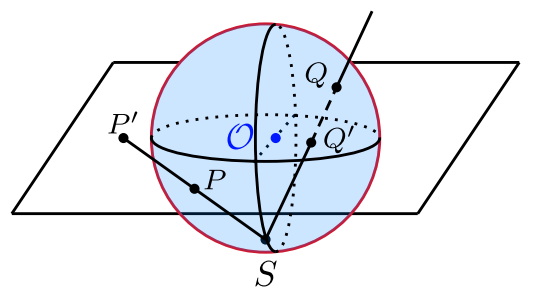

Fig. 2. Schematic representation of the stereographic projections of the unit sphere $S^{2}$ from the south pole $S$ (points $P$ and $Q$ are projected to $P^{\prime}$ and $Q^{\prime}$, respectively).

$$
\begin{gathered}
\hat{\boldsymbol{s}}(\boldsymbol{x})=\hat{\boldsymbol{e}}_{r}=\frac{1}{1+|\boldsymbol{x}|^{2}}\left(\begin{array}{c}
2 x_{1} \\
2 x_{2} \\
1-|\boldsymbol{x}|^{2}
\end{array}\right), \\
\hat{\boldsymbol{\imath}}\left(\boldsymbol{y}_{1}\right)=\frac{1}{1+\left|\boldsymbol{y}_{1}\right|^{2}}\left(\begin{array}{c}
2 y_{11} \\
2 y_{12} \\
1-\left|\boldsymbol{y}_{1}\right|^{2}
\end{array}\right), \\
\hat{\boldsymbol{t}}\left(\boldsymbol{y}_{2}\right)=\frac{1}{1+\left|\boldsymbol{y}_{2}\right|^{2}}\left(\begin{array}{c}
2 y_{21} \\
2 y_{22} \\
1-\left|\boldsymbol{y}_{2}\right|^{2}
\end{array}\right) .
\end{gathered}
$$

We represent the incoming rays $\hat{\boldsymbol{s}}$, intermediate rays $\hat{\boldsymbol{\imath}}$, and outgoing rays $\hat{\boldsymbol{t}}$ using stereographic projections from the south pole $(0,0,-1)$ of $S^{2}$ onto the plane $z=0$, as drawn schematically in Fig. 2. The stereographic projections in Eq. (3) are undefined at the south pole, and we consider $s_{3}, \iota_{3}, t_{3} \neq-1$ and $0 \leq \phi, \psi_{1,2}<\pi$, i.e., the rays are assumed not to be directed downwards parallel to the $z$ axis, and the stereographic projection is always defined. Transforming to stereographic coordinates, we obtain a bounded source domain defined on a plane. For more details, see [6].

We define our source domain $\mathcal{X}$ as the supporting domain of $\tilde{f}(\boldsymbol{x})=f(\phi(\boldsymbol{x}), \theta(\boldsymbol{x}))$, and our target domain $\mathcal{Y}_{2}$ as the image under the mapping $\boldsymbol{m}$, i.e., $\mathcal{Y}_{2}=\boldsymbol{m}(\mathcal{X})$, and we introduce $\tilde{g}\left(\boldsymbol{y}_{2}\right)=g\left(\psi_{2}\left(\boldsymbol{y}_{2}\right), \chi_{2}\left(\boldsymbol{y}_{2}\right)\right)$. We refer to $\boldsymbol{m}: \mathcal{X} \rightarrow \mathcal{Y}_{2}$ as the composite map $\boldsymbol{y}_{2}=\boldsymbol{m}(\boldsymbol{x})$ from the source set of stereographic coordinates $\mathcal{X}$ to the target set of stereographic coordinates $\mathcal{Y}_{2}$. It is a composition of two mappings and can be written as $\boldsymbol{m}=\left(\boldsymbol{m}_{2} \circ \boldsymbol{m}_{1}\right)(\boldsymbol{x})$, where we refer to $\boldsymbol{m}_{1}: \mathcal{X} \rightarrow \mathcal{Y}_{1}$ as the intermediate map $\boldsymbol{y}_{1}=\boldsymbol{m}_{1}(\boldsymbol{x})$ from $\mathcal{X}$ to the image under the mapping $\boldsymbol{m}_{1}$, i.e., $\mathcal{Y}_{1}=\boldsymbol{m}_{1}(\mathcal{X})$, and we refer to $\boldsymbol{m}_{2}: \mathcal{Y}_{1} \rightarrow \mathcal{Y}_{2}$ as the successive map $\boldsymbol{y}_{2}=\boldsymbol{m}_{2}\left(\boldsymbol{y}_{1}\right)$ from $\mathcal{Y}_{1}$ to the image under the mapping $\boldsymbol{m}_{2}$, i.e., $\mathcal{Y}_{2}=\boldsymbol{m}_{2}\left(\mathcal{Y}_{1}\right)=\left(\boldsymbol{m}_{2} \circ \boldsymbol{m}_{1}\right)(\mathcal{X})$.

\section{A. Generating-Function Approach}

In the next two sections, we use Hamilton's characteristic functions to derive the generating functions. In Section 2.A.1, we derive the generating function for the first surface. In Section 2.A.2, we derive the generating function for the second surface.

\section{First Freeform Surface}

Figure 3 illustrates an incident ray propagating in the direction $\hat{\boldsymbol{s}}$, which intercepts the freeform lens surface $\mathcal{L}_{1}$ at point $P_{1}$ and refracts in the direction of the unit vector $\hat{\imath}$.

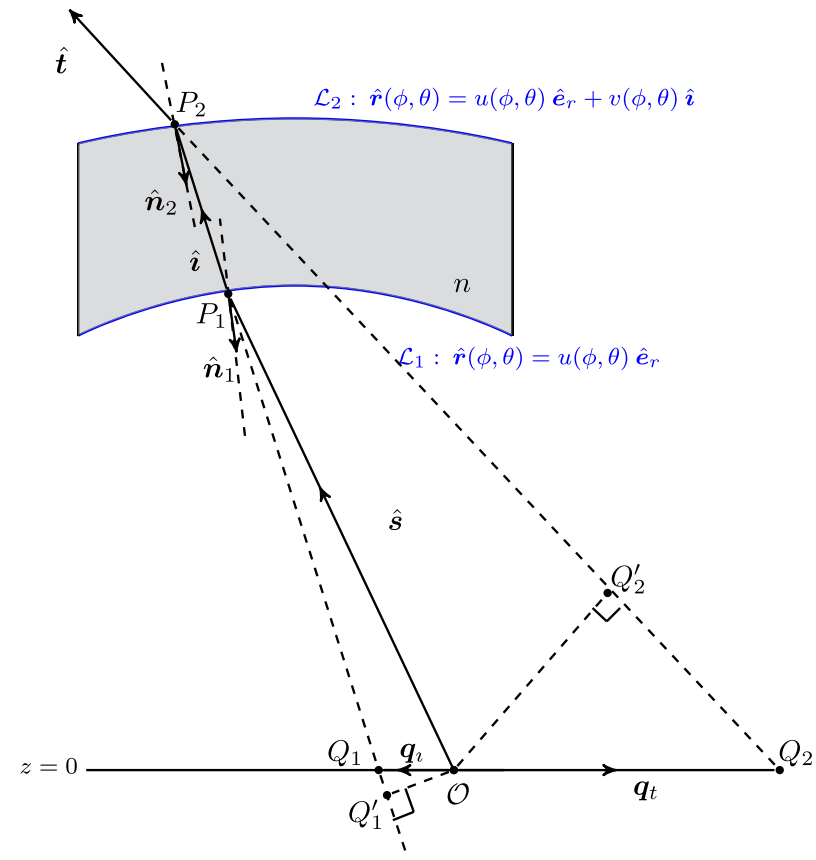

Fig. 3. Sketch of lens with two freeform surfaces.

The position and direction coordinates of the ray $\hat{\boldsymbol{s}}$ at $z=0$ are given by the two vectors $\boldsymbol{q}_{s}=\mathbf{0}$ and $\boldsymbol{p}_{s}=\left(s_{1}, s_{2}\right)$, respectively. The position and direction coordinates of the ray $\hat{\imath}$ at $z=0$ are given by $\boldsymbol{q}_{\iota}$ and $\boldsymbol{p}_{\iota}=\left(n \iota_{1}, n \iota_{2}\right)$, respectively. Note that the position vector of point $P_{1}$ is given by $(u(\hat{\boldsymbol{s}}) \hat{\boldsymbol{s}})=\left(u(\hat{\boldsymbol{s}}) \boldsymbol{p}_{s}, u(\hat{\boldsymbol{s}}) s_{3}\right)$.

The point characteristic $V_{1}$ between point $\mathcal{O}\left(\boldsymbol{q}_{s}, 0\right)$ on the source plane and $Q_{1}\left(\boldsymbol{q}_{l}, 0\right)$ (virtual image of the first surface) is given by

$$
\begin{aligned}
V_{1}\left(\boldsymbol{q}_{s}, \boldsymbol{q}_{l}\right) & =u(\hat{\boldsymbol{s}})-n d\left(P_{1}, Q_{1}\right), \\
d\left(P_{1}, Q_{1}\right) & =\sqrt{\left|\boldsymbol{q}_{l}-u(\hat{\boldsymbol{s}}) \boldsymbol{p}_{s}\right|^{2}+\left(u(\hat{\boldsymbol{s}}) s_{3}\right)^{2}},
\end{aligned}
$$

where $n d\left(P_{1}, Q_{1}\right)$ denotes the OPL between $P_{1}$ and $Q_{1}$, which is equal to the Euclidean distance times $n$.

To derive a generating function, we introduce Hamilton's angular characteristic $T_{1}\left(\boldsymbol{p}_{s}, \boldsymbol{p}_{l}\right)$ as the OPL from $\mathcal{O}$ to $Q_{1}^{\prime}$ in Fig. 3, and show it is independent of $\boldsymbol{p}_{s}$ [41]. Hamilton's angular characteristic for the first surface, which depends on the direction coordinate of the source ray and the intermediate ray, is given by

$$
T_{1}\left(\boldsymbol{p}_{s}, \boldsymbol{p}_{\imath}\right)=V_{1}\left(\boldsymbol{q}_{s}, \boldsymbol{q}_{\imath}\right)+\boldsymbol{q}_{s} \cdot \boldsymbol{p}_{s}-\boldsymbol{q}_{\imath} \cdot \boldsymbol{p}_{\imath} .
$$

The position coordinate $\boldsymbol{q}_{s}$ at the source plane and position coordinate $\boldsymbol{q}_{\imath}$ are given as (p. 105, [41])

$$
\boldsymbol{q}_{s}=\frac{\partial T_{1}}{\partial \boldsymbol{p}_{s}}=\mathbf{0}, \quad \boldsymbol{q}_{\iota}=-\frac{\partial T_{1}}{\partial \boldsymbol{p}_{\iota}} .
$$

By the first equation, we conclude that the angular characteristic $T_{1}$ is independent of the direction coordinate $\boldsymbol{p}_{s}$; thus,

$$
T_{1}\left(\boldsymbol{p}_{t}\right)=V_{1}\left(\boldsymbol{q}_{s}, \boldsymbol{q}_{l}\right)-\boldsymbol{q}_{\imath} \cdot \boldsymbol{p}_{l}=u(\hat{\boldsymbol{s}})-n d\left(P_{1}, Q_{1}\right)-\boldsymbol{q}_{\imath} \cdot \boldsymbol{p}_{l} \cdot
$$

We can derive 


$$
\boldsymbol{p}_{\iota}=n \frac{u(\hat{\boldsymbol{s}}) \boldsymbol{p}_{s}-\boldsymbol{q}_{\iota}}{d\left(P_{1}, Q_{1}\right)}, \quad \iota_{3}=\frac{u(\hat{\boldsymbol{s}}) s_{3}}{d\left(P_{1}, Q_{1}\right)},
$$

and, using Eq. (5) and writing $u=u(\hat{\boldsymbol{s}})$, we obtain

$$
\begin{aligned}
T_{1}\left(\boldsymbol{p}_{\iota}\right)= & u-\frac{n}{d\left(P_{1}, Q_{1}\right)}\left[\left|u \boldsymbol{p}_{s}-\boldsymbol{q}_{\iota}\right|^{2}\right. \\
& \left.+\boldsymbol{q}_{\iota} \cdot\left(u \boldsymbol{p}_{s}-\boldsymbol{q}_{l}\right)+\left(u s_{3}\right)^{2}\right] \\
= & u-\frac{n}{d\left(P_{1}, Q_{1}\right)}\left[\left(u \boldsymbol{p}_{s}-\boldsymbol{q}_{\iota}\right) \cdot u \boldsymbol{p}_{s}\right]-n \iota_{3}\left(u s_{3}\right) .
\end{aligned}
$$

Rearranging terms and substituting $\boldsymbol{p}_{\iota}$ from Eq. (9) gives

$$
\begin{aligned}
T_{1}\left(\boldsymbol{p}_{\iota}\right) & =u-n u\left[\frac{1}{d\left(P_{1}, Q_{1}\right)}\left(u \boldsymbol{p}_{s}-\boldsymbol{q}_{\iota}\right) \cdot \boldsymbol{p}_{s}+s_{3} \iota_{3}\right] \\
& =u-n u\left(\boldsymbol{p}_{\iota} / n \cdot \boldsymbol{p}_{s}+s_{3} \iota_{3}\right) \\
& =u(1-n \hat{\boldsymbol{s}} \cdot \hat{\boldsymbol{\imath}}) .
\end{aligned}
$$

Solving this equation for $u(\hat{\boldsymbol{s}})$, we obtain

$$
u(\hat{\boldsymbol{s}})=\frac{T_{1}\left(\boldsymbol{p}_{\iota}\right)}{1-n \hat{\boldsymbol{s}} \cdot \hat{\boldsymbol{\imath}}} .
$$

Changing to stereographic coordinates using Eqs. (4a) and (4b) gives

$$
u(\boldsymbol{x})=T_{1}\left(\boldsymbol{p}_{\iota}\right)\left(1-n+\frac{2 n\left|\boldsymbol{x}-\boldsymbol{y}_{1}\right|^{2}}{\left(1+|\boldsymbol{x}|^{2}\right)\left(1+\left|\boldsymbol{y}_{1}\right|^{2}\right)}\right)^{-1},
$$

where, for ease of notation, we continue to use the variable $u$ to represent the optical surface, but now as a function of $\boldsymbol{x}$. We construct the generating function $G_{1}$ from the relation $u(\boldsymbol{x})=G_{1}\left(\boldsymbol{x}, \boldsymbol{y}_{1}, z\right)$, with $z=T_{1}\left(\boldsymbol{p}_{l}\right)$, as

$$
G_{1}\left(\boldsymbol{x}, \boldsymbol{y}_{1}, z\right)=z\left(1-n+\frac{2 n\left|\boldsymbol{x}-\boldsymbol{y}_{1}\right|^{2}}{\left(1+|\boldsymbol{x}|^{2}\right)\left(1+\left|\boldsymbol{y}_{1}\right|^{2}\right)}\right)^{-1} .
$$

Note that $z=T_{1}\left(\boldsymbol{p}_{l}\right)$ is dependent on the outgoing ray $\hat{\boldsymbol{\imath}}$, and hence $z=z\left(\boldsymbol{y}_{1}\right)$ is a function of $\boldsymbol{y}_{1}$.

\section{Second Freeform Surface}

At $z=0$, the position and direction coordinates of the ray $\hat{\boldsymbol{t}}$ are given by $\boldsymbol{q}_{t}$ and $\boldsymbol{p}_{t}=\left(t_{1}, t_{2}\right)$, respectively. Note that the position vector of point $P_{2}$ is given by

$$
(u(\hat{\boldsymbol{s}}) \hat{\boldsymbol{s}}+v(\hat{\boldsymbol{s}}) \hat{\boldsymbol{\imath}})=\left(u(\hat{\boldsymbol{s}}) \boldsymbol{p}_{s}+v(\hat{\boldsymbol{s}}) \boldsymbol{p}_{l} / n, u(\hat{\boldsymbol{s}}) s_{3}+v(\hat{\boldsymbol{s}}) \iota_{3}\right) .
$$

The total point characteristic between point $\mathcal{O}\left(\boldsymbol{q}_{s}, 0\right)$ on the source plane and $Q_{2}\left(\boldsymbol{q}_{t}, 0\right)$ (virtual image of the second surface) is given by

$$
\begin{aligned}
V\left(\boldsymbol{q}_{s}, \boldsymbol{q}_{t}\right) & =u(\hat{\boldsymbol{s}})+n d\left(P_{1}, P_{2}\right)-d\left(P_{2}, Q_{2}\right), \\
d\left(P_{2}, Q_{2}\right) & =\sqrt{\left|\left(u(\hat{\boldsymbol{s}}) \boldsymbol{p}_{s}+v(\hat{\boldsymbol{s}}) \boldsymbol{p}_{l} / n\right)-\boldsymbol{q}_{t}\right|^{2}+\left(u(\hat{\boldsymbol{s}}) s_{3}+v(\hat{\boldsymbol{s}}) \iota_{3}\right)^{2}},
\end{aligned}
$$

where $n d\left(P_{1}, P_{2}\right)=n v(\hat{\boldsymbol{s}})$ denotes the OPL between $P_{1}$ and $P_{2}$, and $d\left(P_{2}, Q_{2}\right)$ is the Euclidean distance between $P_{2}$ and $Q_{2}$.
As in the previous section, we introduce Hamilton's angular characteristic $T\left(\boldsymbol{p}_{s}, \boldsymbol{p}_{t}\right)$ as the OPL from $\mathcal{O}$ to $Q_{2}^{\prime}$ in Fig. 3 , and show it is independent of $\boldsymbol{p}_{s}$ [41]. Hamilton's angular characteristic for the second surface, which depends on the direction of the source ray and the final ray, is given by

$$
T\left(\boldsymbol{p}_{s}, \boldsymbol{p}_{t}\right)=V\left(\boldsymbol{q}_{s}, \boldsymbol{q}_{t}\right)+\boldsymbol{q}_{s} \cdot \boldsymbol{p}_{s}-\boldsymbol{q}_{t} \cdot \boldsymbol{p}_{t} .
$$

The position coordinate $\boldsymbol{q}_{s}$ at the source plane and position coordinate $\boldsymbol{q}_{t}$ are given as (p. 105, [41])

$$
\boldsymbol{q}_{s}=\frac{\partial T}{\partial \boldsymbol{p}_{s}}=\mathbf{0}, \quad \boldsymbol{q}_{t}=-\frac{\partial T}{\partial \boldsymbol{p}_{t}} .
$$

By the former equation, we conclude that the angular characteristic $T$ is independent of the direction coordinate $\boldsymbol{p}_{s}$, and

$$
\begin{aligned}
T\left(\boldsymbol{p}_{t}\right) & =V\left(\boldsymbol{q}_{s}, \boldsymbol{q}_{t}\right)-\boldsymbol{q}_{t} \cdot \boldsymbol{p}_{t} \\
& =u(\hat{\boldsymbol{s}})+n v(\hat{\boldsymbol{s}})-d\left(P_{2}, Q_{2}\right)-\boldsymbol{q}_{t} \cdot \boldsymbol{p}_{t} .
\end{aligned}
$$

We can derive

$$
\boldsymbol{p}_{t}=\frac{u(\hat{\boldsymbol{s}}) \boldsymbol{p}_{s}+v(\hat{\boldsymbol{s}}) \boldsymbol{p}_{l} / n-\boldsymbol{q}_{t}}{d\left(P_{2}, Q_{2}\right)}, \quad t_{3}=\frac{u(\hat{\boldsymbol{s}}) s_{3}+v(\hat{\boldsymbol{s}}) \iota_{3}}{d\left(P_{2}, Q_{2}\right)},
$$

and, using Eq. (15) and writing $u=u(\hat{\boldsymbol{s}})$ and $v=v(\hat{\boldsymbol{s}})$,

$$
\begin{aligned}
T\left(\boldsymbol{p}_{t}\right)= & u+n v-\frac{1}{d\left(P_{2}, Q_{2}\right)}\left[\left|u \boldsymbol{p}_{s}+v \boldsymbol{p}_{l} / n-\boldsymbol{q}_{t}\right|^{2}\right. \\
& \left.+\boldsymbol{q}_{t} \cdot\left(u \boldsymbol{p}_{s}+v \boldsymbol{p}_{l} / n-\boldsymbol{q}_{t}\right)+\left(u s_{3}+v \iota_{3}\right)^{2}\right] \\
= & u+n v-\frac{1}{d\left(P_{2}, Q_{2}\right)} \\
& \times\left[\left(u \boldsymbol{p}_{s}+v \boldsymbol{p}_{l} / n-\boldsymbol{q}_{t}\right) \cdot\left(u \boldsymbol{p}_{s}+v \boldsymbol{p}_{l} / n\right)\right] \\
& -t_{3}\left(u s_{3}+v \iota_{3}\right) .
\end{aligned}
$$

Using Eq. (19) and reordering terms gives

$$
\begin{aligned}
T\left(\boldsymbol{p}_{t}\right)= & u+n v-\left[\frac{1}{d\left(P_{2}, Q_{2}\right)}\left(u \boldsymbol{p}_{s}+v \boldsymbol{p}_{l} / n-\boldsymbol{q}_{t}\right) .\right. \\
& \left.\left(u \boldsymbol{p}_{s}+v \boldsymbol{p}_{l} / n\right)+u s_{3} t_{3}+v \iota_{3} t_{3}\right] \\
= & u+n v-\left[\boldsymbol{p}_{t} \cdot\left(u \boldsymbol{p}_{s}+v \boldsymbol{p}_{l} / n\right)+u s_{3} t_{3}+v \iota_{3} t_{3}\right] \\
= & u(1-\hat{\boldsymbol{s}} \cdot \hat{\boldsymbol{t}})+v(n-\hat{\boldsymbol{\imath}} \cdot \hat{\boldsymbol{t}}) .
\end{aligned}
$$

Hence, we arrive at $T\left(\boldsymbol{p}_{t}\right)=u(\hat{\boldsymbol{s}})(1-\hat{\boldsymbol{s}} \cdot \hat{\boldsymbol{t}})+v(\hat{\boldsymbol{s}})(n-\hat{\boldsymbol{\imath}} \cdot \hat{\boldsymbol{t}})$.

Note that we can also derive that $T\left(\boldsymbol{p}_{t}\right)$ is the sum of $T_{1}\left(\boldsymbol{p}_{l}\right)$ and $T_{2}\left(\boldsymbol{p}_{l}, \boldsymbol{p}_{t}\right)$, where $T_{2}\left(\boldsymbol{p}_{l}, \boldsymbol{p}_{t}\right)$ is the angular characteristic from $Q_{1}$ to $Q_{2}$. Indeed, the expression for $T_{2}\left(\boldsymbol{p}_{\iota}, \boldsymbol{p}_{t}\right)$ is

$$
\begin{aligned}
T_{2}\left(\boldsymbol{p}_{\imath}, \boldsymbol{p}_{t}\right) & =V_{2}\left(\boldsymbol{q}_{\imath}, \boldsymbol{q}_{t}\right)+\boldsymbol{q}_{\imath} \cdot \boldsymbol{p}_{\imath}-\boldsymbol{q}_{t} \cdot \boldsymbol{p}_{t} \\
& =n d\left(P_{1}, Q_{1}\right)+n v(\hat{\boldsymbol{s}})-d\left(P_{2}, Q_{2}\right)+\boldsymbol{q}_{\imath} \cdot \boldsymbol{p}_{\iota}-\boldsymbol{q}_{t} \cdot \boldsymbol{p}_{t},
\end{aligned}
$$

and adding Eq. (8), we see that $\boldsymbol{q}_{\iota} \cdot \boldsymbol{p}_{\iota}$ and $n d\left(P_{1}, Q_{1}\right)$ cancel and that 


$$
T_{1}\left(\boldsymbol{p}_{t}\right)+T_{2}\left(\boldsymbol{p}_{t}, \boldsymbol{p}_{t}\right)=T\left(\boldsymbol{p}_{t}\right)=u(\hat{\boldsymbol{s}})+n v(\hat{\boldsymbol{s}})-d\left(P_{2}, Q_{2}\right)-\boldsymbol{q}_{t} \cdot \boldsymbol{p}_{t},
$$

as in Eq. (14).

Solving Eq. (20) for $v(\hat{\boldsymbol{s}})$, we obtain

$$
v(\hat{\boldsymbol{s}})=\frac{T\left(\boldsymbol{p}_{t}\right)-u(\hat{\boldsymbol{s}})(1-\hat{\boldsymbol{s}} \cdot \hat{\boldsymbol{t}})}{n-\hat{\boldsymbol{\imath}} \cdot \hat{\boldsymbol{t}}} .
$$

Changing to stereographic coordinates using Eq. (3) gives

$$
\begin{aligned}
v(\boldsymbol{x})= & \left(T\left(\boldsymbol{p}_{t}\right)-u(\boldsymbol{x}) \frac{2\left|\boldsymbol{x}-\boldsymbol{y}_{2}\right|^{2}}{\left(1+|\boldsymbol{x}|^{2}\right)\left(1+\left|\boldsymbol{y}_{2}\right|^{2}\right.}\right) \\
& \times\left(n-1+\frac{2\left|\boldsymbol{y}_{1}-\boldsymbol{y}_{2}\right|^{2}}{\left(1+\left|\boldsymbol{y}_{1}\right|^{2}\right)\left(1+\left|\boldsymbol{y}_{2}\right|^{2}\right)}\right)^{-1},
\end{aligned}
$$

where we use the variable $v$ to represent the optical surface, but now as a function of $\boldsymbol{x}$. Next, we construct the generating function $G_{2}$ from the relation $v(\boldsymbol{x})=G_{2}\left(\boldsymbol{x}, \boldsymbol{y}_{2}, z\right)$, with $z=T\left(\boldsymbol{p}_{t}\right)$, as

$$
\begin{aligned}
G_{2}\left(\boldsymbol{x}, \boldsymbol{y}_{2}, z\right)= & \left(z-u(\boldsymbol{x}) \frac{2\left|\boldsymbol{x}-\boldsymbol{y}_{2}\right|^{2}}{\left(1+|\boldsymbol{x}|^{2}\right)\left(1+\left|\boldsymbol{y}_{2}\right|\right)^{2}}\right) \\
& \times\left(n-1+\frac{2\left|\boldsymbol{y}_{1}-\boldsymbol{y}_{2}\right|^{2}}{\left(1+\left|\boldsymbol{y}_{1}\right|^{2}\right)\left(1+\left|\boldsymbol{y}_{2}\right|^{2}\right)}\right)^{-1} .
\end{aligned}
$$

Note that $z=T\left(\boldsymbol{p}_{t}\right)$ is dependent on the outgoing ray $\hat{\boldsymbol{t}}$, and hence $z=z\left(\boldsymbol{y}_{2}\right)$ is a function of $\boldsymbol{y}_{2}$.

\section{B. G-Convex Solutions}

In this paper, we first consider the generating function $G_{1}$ in Eq. (13) to compute the first surface $u(\boldsymbol{x})$ and intermediate mapping $\boldsymbol{m}_{1}$, and subsequently the generating function $G_{2}$ in Eq. (25) to compute $v(\boldsymbol{x})$ and the composite mapping $\boldsymbol{m}$.

\section{First Freeform Surface}

The equation $G_{1}\left(\boldsymbol{x}, \boldsymbol{y}_{1}, z\right)=u(\boldsymbol{x})$ with $G_{1}$ given in Eq. (13) and $z=z\left(\boldsymbol{y}_{1}\right)$ has many solutions for $u(\boldsymbol{x})$. For the computation of the first surface, we let $\boldsymbol{y}=\boldsymbol{y}_{1}, u_{1}(\boldsymbol{x})=u(\boldsymbol{x})$, and $u_{2}(\boldsymbol{y})=z(\boldsymbol{y})$. Then we have

$$
\forall \boldsymbol{x} \in \mathcal{X}, \forall \boldsymbol{y} \in \mathcal{Y}: \quad u_{1}(\boldsymbol{x})=G_{1}\left(\boldsymbol{x}, \boldsymbol{y}, u_{2}(\boldsymbol{y})\right) .
$$

We define $H_{1}\left(\boldsymbol{x}, \boldsymbol{y}, G_{1}\left(\boldsymbol{x}, \boldsymbol{y}, u_{2}(\boldsymbol{y})\right)\right)=u_{2}(\boldsymbol{y})$ as the unique inverse of $G_{1}$ for a given $\boldsymbol{x} \in \mathcal{X}$ and $\boldsymbol{y} \in \mathcal{Y}$, assuming a unique inverse exists. Then, for all $\boldsymbol{x} \in \mathcal{X}, \boldsymbol{y} \in \mathcal{Y}$, we have

$$
u_{1}(\boldsymbol{x})=G_{1}\left(\boldsymbol{x}, \boldsymbol{y}, u_{2}(\boldsymbol{y})\right) \Leftrightarrow u_{2}(\boldsymbol{y})=H_{1}\left(\boldsymbol{x}, \boldsymbol{y}, u_{1}(\boldsymbol{x})\right),
$$

i.e., for fixed $\boldsymbol{x}, \boldsymbol{y}$, we have that $G_{1}(\boldsymbol{x}, \boldsymbol{y}, \cdot)$ and $H_{1}(\boldsymbol{x}, \boldsymbol{y}, \cdot)$ are each other's inverses. Hence, for the existence of a unique inverse, we require that for all $\boldsymbol{x} \in \mathcal{X}, \boldsymbol{y} \in \mathcal{Y}, z \in \mathbb{R}$, we have $G_{1, z}>0$ or $G_{1, z}<0$, since $G_{1}$ should be injective w.r.t. the third argument $\left(G_{1, z}=0\right.$ is possible at an isolated point); see Section 2.C.

For the first freeform surface, the function $H_{1}$ is the angular characteristic $T_{1}\left(\boldsymbol{p}_{l}\right)$ rewritten in stereographic coordinates, cf.
Eqs. (12) and (13), i.e.,

$$
H_{1}(\boldsymbol{x}, \boldsymbol{y}, z)=z\left(1-n+\frac{2 n|\boldsymbol{x}-\boldsymbol{y}|^{2}}{\left(1+|\boldsymbol{x}|^{2}\right)\left(1+|\boldsymbol{y}|^{2}\right)}\right) .
$$

Energy conservation: by transferring the light from source to intermediate target, we require that all light from the source ends up at the intermediate target and energy is conserved, i.e.,

$$
\int_{\mathcal{A}} f(\phi, \theta) \mathrm{d} \boldsymbol{S}(\phi, \theta)=\int_{\hat{\imath}(\mathcal{A})} h\left(\psi_{1}, \chi_{1}\right) \mathrm{d} \boldsymbol{S}\left(\psi_{1}, \chi_{1}\right),
$$

for an arbitrary set $\mathcal{A} \subset S^{2}$ and image set $\hat{\imath}(\mathcal{A}) \subset S^{2}$. Note that this image set corresponds to the far-field approximation. If we substitute $\hat{\boldsymbol{s}}=\hat{\boldsymbol{s}}(\boldsymbol{x})$ and $\hat{\boldsymbol{\imath}}=\hat{\boldsymbol{\imath}}\left(\boldsymbol{y}_{1}\right)$ from Eqs. (4a) and (4b), we can write Eq. (29) as

$\int_{\boldsymbol{x}(\mathcal{A})} \tilde{f}(\boldsymbol{x})\left|\frac{\partial \hat{\boldsymbol{s}}}{\partial x_{1}} \times \frac{\partial \hat{\boldsymbol{s}}}{\partial x_{2}}\right| \mathrm{d} \boldsymbol{x}=\int_{\boldsymbol{y}_{1}(\hat{\imath}(\mathcal{A}))} \tilde{h}\left(\boldsymbol{y}_{1}\right)\left|\frac{\partial \hat{\boldsymbol{\imath}}}{\partial y_{11}} \times \frac{\partial \hat{\boldsymbol{\imath}}}{\partial y_{12}}\right| \mathrm{d} \boldsymbol{y}_{1}$,

where $\tilde{f}(\boldsymbol{x})=f(\phi, \theta)$, and $\tilde{h}\left(\boldsymbol{y}_{1}\right)=h\left(\psi_{1}, \chi_{1}\right)$. We can derive that

$$
\left|\frac{\partial \hat{\boldsymbol{s}}}{\partial x_{1}} \times \frac{\partial \hat{\boldsymbol{s}}}{\partial x_{2}}\right|=\frac{4}{\left(1+|\boldsymbol{x}|^{2}\right)^{2}}, \quad \quad\left|\frac{\partial \hat{\boldsymbol{\imath}}}{\partial y_{11}} \times \frac{\partial \hat{\boldsymbol{\imath}}}{\partial y_{12}}\right|=\frac{4}{\left(1+\left|\boldsymbol{y}_{1}\right|^{2}\right)^{2}} .
$$

Substituting Eq. (31) and the mapping $\boldsymbol{y}_{1}=\boldsymbol{m}_{1}(\boldsymbol{x})$ into the energy conservation relation Eq. (30) gives

$$
\begin{aligned}
& \int_{\boldsymbol{x}(\mathcal{A})} \tilde{f}(\boldsymbol{x}) \frac{4}{\left(1+|\boldsymbol{x}|^{2}\right)^{2}} \mathrm{~d} \boldsymbol{x} \\
& =\int_{\boldsymbol{x}(\mathcal{A})} \tilde{h}\left(\boldsymbol{m}_{1}(\boldsymbol{x})\right) \frac{4}{\left(1+\left|\boldsymbol{m}_{1}(\boldsymbol{x})\right|^{2}\right)^{2}} \operatorname{det}\left(\mathrm{Dm}_{1}(\boldsymbol{x})\right) \mathrm{d} \boldsymbol{x},
\end{aligned}
$$

where we omit the absolute value sign of the determinant and restrict ourselves to a positive Jacobian of the mapping. We can rewrite Eq. (32) to the generalized Monge-Ampère equation

$$
\begin{aligned}
\operatorname{det}\left(\operatorname{D} m_{1}(\boldsymbol{x})\right) & =\frac{\tilde{f}(\boldsymbol{x})}{\tilde{h}\left(\boldsymbol{m}_{1}(\boldsymbol{x})\right)} \frac{\left(1+\left|\boldsymbol{m}_{1}(\boldsymbol{x})\right|^{2}\right)^{2}}{\left(1+|\boldsymbol{x}|^{2}\right)^{2}} \\
& =F_{1}\left(\boldsymbol{x}, \boldsymbol{m}_{1}(\boldsymbol{x}), u_{1}(\boldsymbol{x})\right),
\end{aligned}
$$

where we introduce $F_{1}\left(\boldsymbol{x}, \boldsymbol{m}_{1}(\boldsymbol{x}), u_{1}(\boldsymbol{x})\right)$ to denote the total right-hand side with $u_{1}(\boldsymbol{x})=u(\boldsymbol{x})$. The right-hand side also depends on $u_{1}(\boldsymbol{x})=u(\boldsymbol{x})$ since the mapping $\boldsymbol{y}_{1}=\boldsymbol{m}_{1}(\boldsymbol{x})=\boldsymbol{m}_{1}\left(\boldsymbol{x}, u_{1}(\boldsymbol{x}), \nabla u_{1}(\boldsymbol{x})\right)$ is a function of $u_{1}$ and its gradient. An explanation will be given in Section 2.C.

We define the corresponding transport boundary condition to Eq. (33a) as

$$
\boldsymbol{m}_{1}(\partial \mathcal{X})=\partial \mathcal{Y}_{1},
$$

stating that all light from the boundary of the source $\mathcal{X}$ is mapped to the boundary of the target $\mathcal{Y}_{1}[3,42]$.

We use the algorithm in [1] to compute $\boldsymbol{y}_{1}=\boldsymbol{m}_{1}(\boldsymbol{x})$ and $u_{1}(\boldsymbol{x})=u(\boldsymbol{x})$. 


\section{Second Freeform Surface}

After computing $u_{1}(\boldsymbol{x})=u(\boldsymbol{x})$ and $\boldsymbol{y}_{1}(\boldsymbol{x})$ as functions of $\boldsymbol{x}$ for the first surface, we proceed to compute $v(\boldsymbol{x})$ and the composite mapping $\boldsymbol{y}_{2}=\boldsymbol{m}(\boldsymbol{x})$. The equation $G_{2}\left(\boldsymbol{x}, \boldsymbol{y}_{2}, z\right)=v(\boldsymbol{x})$, with $G_{2}$ given in Eq. (25) and $z=z\left(\boldsymbol{y}_{2}\right)$, has many solutions for $v(\boldsymbol{x})$. For the second freeform surface, we let $\boldsymbol{y}=\boldsymbol{y}_{2}$, $u_{1}(\boldsymbol{x})=v(\boldsymbol{x})$, and $u_{2}(\boldsymbol{y})=z(\boldsymbol{y})$. Then the unique inverse function $H_{2}(\boldsymbol{x}, \boldsymbol{y}, z)$, with $z=u_{1}(\boldsymbol{x})$, is the angular characteristic $T\left(\boldsymbol{p}_{t}\right)$ rewritten in stereographic coordinates, cf. Eqs. (24) and (25), as

$$
\begin{aligned}
H_{2}(\boldsymbol{x}, \boldsymbol{y}, z)= & u(\boldsymbol{x})\left(\frac{2|\boldsymbol{x}-\boldsymbol{y}|^{2}}{\left(1+|\boldsymbol{x}|^{2}\right)\left(1+|\boldsymbol{y}|^{2}\right)}\right) \\
& +z\left(n-1+\frac{2\left|\boldsymbol{y}_{1}(\boldsymbol{x})-\boldsymbol{y}\right|^{2}}{\left(1+\left|\boldsymbol{y}_{1}(\boldsymbol{x})\right|^{2}\right)\left(1+|\boldsymbol{y}|^{2}\right)}\right) .
\end{aligned}
$$

Energy conservation: by transferring the light from the source to the final target, we require that all light from the source ends up at the target and energy is conserved, i.e.,

$$
\int_{\mathcal{A}} f(\phi, \theta) \mathrm{d} \boldsymbol{S}(\phi, \theta)=\int_{\hat{\boldsymbol{t}}(\mathcal{A})} g\left(\psi_{2}, \chi_{2}\right) \mathrm{d} \boldsymbol{S}\left(\psi_{2}, \chi_{2}\right),
$$

for an arbitrary set $\mathcal{A} \subset S^{2}$ and image set $\hat{\boldsymbol{t}}(\mathcal{A}) \subset S^{2}$. Following the arguments of the previous section, if we substitute $\hat{\boldsymbol{s}}=\hat{\boldsymbol{s}}(\boldsymbol{x})$ and $\hat{\boldsymbol{t}}=\hat{\boldsymbol{t}}\left(\boldsymbol{y}_{2}\right)$ from Eqs. (4a) and (4c), and substitute the mapping $\boldsymbol{y}_{2}=\boldsymbol{m}(\boldsymbol{x})=\boldsymbol{m}_{2}\left(\boldsymbol{m}_{1}(\boldsymbol{x})\right)$, we obtain the generalized Monge-Ampère equation

$$
\begin{aligned}
\operatorname{det}(\operatorname{Dm}(\boldsymbol{x})) & =\frac{\tilde{f}(\boldsymbol{x})}{\tilde{g}(\boldsymbol{m}(\boldsymbol{x}))} \frac{\left(1+|\boldsymbol{m}(\boldsymbol{x})|^{2}\right)^{2}}{\left(1+|\boldsymbol{x}|^{2}\right)^{2}} \\
& =F_{2}\left(\boldsymbol{x}, \boldsymbol{m}(\boldsymbol{x}), u_{1}(\boldsymbol{x})\right)
\end{aligned}
$$

where we introduce $F_{2}\left(\boldsymbol{x}, \boldsymbol{m}(\boldsymbol{x}), u_{1}(\boldsymbol{x})\right)$ to denote the total right-hand side with $u_{1}(\boldsymbol{x})=v(\boldsymbol{x})$, and we have the transport boundary condition

$$
\boldsymbol{m}(\partial \mathcal{X})=\partial \mathcal{Y}_{2}
$$

Hence, we have a first-order partial differential equation for the composite mapping $\boldsymbol{m}$. We proceed by using the algorithm in [1] to compute $\boldsymbol{y}_{2}=\boldsymbol{m}(\boldsymbol{x})$ and $u_{1}(\boldsymbol{x})=v(\boldsymbol{x})$. The numerical algorithm uses the function $H_{2}$ in Eq. (34) after we have first computed and substituted $u(\boldsymbol{x})$ and the intermediate mapping $\boldsymbol{y}_{1}(\boldsymbol{x})=\boldsymbol{m}_{1}(\boldsymbol{x})$. In the next section, it will be explained how we can use functions $H_{1}$ and $H_{2}$ to find a unique solution $u(\boldsymbol{x})$ and an expression for the intermediate mapping $\boldsymbol{m}_{1}(\boldsymbol{x})$ for the first surface and a unique solution $v(\boldsymbol{x})$ and composite mapping $\boldsymbol{m}(\boldsymbol{x})$ for the second surface, respectively.

\section{Using $\boldsymbol{H}$ to Compute the Mapping and Surface}

For both surfaces, we can find a unique solution by assuming that $u_{1}$ is a G-convex or G-concave function. Recall for the first surface, $G=G_{1}, H=H_{1}, u_{1}(\boldsymbol{x})=u(\boldsymbol{x}), \boldsymbol{y}=\boldsymbol{y}_{1}$, and $u_{2}(\boldsymbol{y})=T\left(\boldsymbol{p}_{\imath}\right)$, and for the second surface, $G=G_{2}, H=H_{2}$, $u_{1}(\boldsymbol{x})=v(\boldsymbol{x}), \boldsymbol{y}=\boldsymbol{y}_{2}$, and $u_{2}(\boldsymbol{y})=T\left(\boldsymbol{p}_{t}\right)$. In this section, we continue in general terms, which applies to both optical surfaces. The function $u_{1}(\boldsymbol{x})$ is $\mathrm{G}$-convex and $u_{2}(\boldsymbol{y})$ is H-concave if

$$
\begin{array}{ll}
\forall \boldsymbol{x} \in \mathcal{X}: & u_{1}(\boldsymbol{x})=\max _{\boldsymbol{y} \in \mathcal{Y}} G\left(\boldsymbol{x}, \boldsymbol{y}, u_{2}(\boldsymbol{y})\right), \\
\forall \boldsymbol{y} \in \mathcal{Y}: & u_{2}(\boldsymbol{y})=\min _{\boldsymbol{x} \in \mathcal{X}} H\left(\boldsymbol{x}, \boldsymbol{y}, u_{1}(\boldsymbol{x})\right),
\end{array}
$$

or $u_{1}(\boldsymbol{x})$ is $\mathrm{G}$-concave and $u_{2}(\boldsymbol{y})$ is H-convex if

$$
\begin{array}{ll}
\forall \boldsymbol{x} \in \mathcal{X}: & u_{1}(\boldsymbol{x})=\min _{\boldsymbol{y} \in \mathcal{Y}} G\left(\boldsymbol{x}, \boldsymbol{y}, u_{2}(\boldsymbol{y})\right), \\
\forall \boldsymbol{y} \in \mathcal{Y}: & u_{2}(\boldsymbol{y})=\max _{\boldsymbol{x} \in \mathcal{X}} H\left(\boldsymbol{x}, \boldsymbol{y}, u_{1}(\boldsymbol{x})\right) .
\end{array}
$$

For the second surface we have the property $G_{z}>0$, cf. Eqs. (23) and (25), since $n-\hat{\boldsymbol{\imath}} \cdot \hat{\boldsymbol{t}}>0$ with $n>1$, which results in the $\mathrm{max} / \mathrm{min}$ pair in Eq. (37) for a G-convex solution $u_{1}$ or in the $\mathrm{min} / \mathrm{max}$ pair in Eq. (38) for a G-concave solution $u_{1}$, as in [1]. For the first surface, this is not necessarily true; see Eqs. (11) and (13). For $G_{z}>0$, we require $1-n \hat{\boldsymbol{s}} \cdot \hat{\boldsymbol{\imath}}>0$. Whether this inequality is satisfied depends on the choice of source and target domains. Otherwise, $G_{z}<0$, which results in a max/max pair for a G-convex solution and $\mathrm{min} / \mathrm{min}$ pair for a G-concave solution, analogous to Eqs. (37) and (38), but this does not have an effect on our numerical method. In our numerical examples in Section 4, we compute a G-concave solution for $u(\mathrm{~min} / \mathrm{min}$ pair), after verifying that $G_{z}<0$ at all points of the domain, and a G-convex solution for $v$ since $G_{z}>0$ ( $\mathrm{max} / \mathrm{min}$ pair). We note that we can also choose other combinations of G-convex and G-concave surfaces for the double freeform lens, which results in different designs.

By the implicit function theorem, the mapping $\boldsymbol{y}=\boldsymbol{m}\left(\boldsymbol{x}, u_{1}(\boldsymbol{x}), \nabla u_{1}(\boldsymbol{x})\right)$ is given implicitly as the critical point of Eq. (37b) or Eq. (38b), i.e.,

$$
\nabla_{\boldsymbol{x}} H\left(\boldsymbol{x}, \boldsymbol{y}, u_{1}(\boldsymbol{x})\right)+H_{z}\left(\boldsymbol{x}, \boldsymbol{y}, u_{1}(\boldsymbol{x})\right) \nabla u_{1}(\boldsymbol{x})=\mathbf{0} .
$$

If $H$ is given by Eq. (28) and $u_{1}(\boldsymbol{x})=u(\boldsymbol{x})$, this equation implicitly defines $\boldsymbol{y}_{1}=\boldsymbol{m}_{1}(\boldsymbol{x})$. If $H$ is given by Eq. (34) and $u_{1}(\boldsymbol{x})=v(\boldsymbol{x})$, this equation implicitly defines $\boldsymbol{y}_{2}=\boldsymbol{m}(\boldsymbol{x})$. In the following, we drop subscripts and simply refer to $\boldsymbol{y}=\boldsymbol{m}(\boldsymbol{x})$ as the mapping corresponding to a general $H$.

For simplicity, since $u_{1}=u_{1}(\boldsymbol{x})$, we define $H^{*}(\boldsymbol{x}, \boldsymbol{y})=$ $H\left(\boldsymbol{x}, \boldsymbol{y}, u_{1}(\boldsymbol{x})\right)$ and rewrite Eq. (39) to the shorter form

$$
\nabla_{\boldsymbol{x}} H^{*}(\boldsymbol{x}, \boldsymbol{y})=\mathbf{0}
$$

and use the implicit function theorem to denote the mapping $\boldsymbol{y}=\boldsymbol{m}(\boldsymbol{x})$ as a function of $\boldsymbol{x}$ only.

A sufficient condition for a minimum in Eq. (37b) or a maximum in Eq. (38b) is for the Hessian matrix $-\mathrm{D}_{\boldsymbol{x} x} H^{*}(\boldsymbol{x}, \boldsymbol{m}(\boldsymbol{x}))=\boldsymbol{P}(\boldsymbol{x})$ to be symmetric negative definite (SND) or symmetric positive definite (SPD), respectively. For an SND matrix $\boldsymbol{P}$, we need $\operatorname{tr}(\boldsymbol{P}) \leq 0$ and $\operatorname{det}(\boldsymbol{P}) \geq 0$. On the other hand, for an SPD matrix, we need $\operatorname{tr}(\boldsymbol{P}) \geq 0$ and $\operatorname{det}(\boldsymbol{P}) \geq 0$. Note that $\boldsymbol{P}$ is symmetric. In this paper, we choose to compute a G-concave (and H-concave) solution $(\mathrm{min} / \mathrm{min}$ pair) for $u$ and a G-convex (and H-concave) solution ( $\mathrm{max} / \mathrm{min}$ pair) for $v$. In both cases, we require $\boldsymbol{P}$ to be SND. 
Substituting $\boldsymbol{y}=\boldsymbol{m}(\boldsymbol{x})$ in Eq. (40) and differentiating again with respect to $\boldsymbol{x}$ gives

$$
\mathrm{D}_{x x} H^{*}(\boldsymbol{x}, \boldsymbol{m}(\boldsymbol{x}))+\mathrm{D}_{x y} H^{*}(\boldsymbol{x}, \boldsymbol{m}(\boldsymbol{x})) \mathrm{D} \boldsymbol{m}(\boldsymbol{x})=\mathbf{0},
$$

where $\mathrm{D}_{x x} H^{*}$ is the Hessian matrix of $H^{*}$ with respect to $\boldsymbol{x}$, $\mathrm{D}_{x y} H^{*}$ is the matrix of mixed second-order partial derivatives with respect to $\boldsymbol{x}$ and $\boldsymbol{y}$, and $\mathrm{D} \boldsymbol{m}(\boldsymbol{x})$ is the $2 \times 2$ Jacobi matrix of $\boldsymbol{m}$ with respect to $\boldsymbol{x}$. Using $-\mathrm{D}_{\boldsymbol{x} x} H^{*}(\boldsymbol{x}, \boldsymbol{m}(\boldsymbol{x}))=\boldsymbol{P}$, we find

$$
\begin{aligned}
\boldsymbol{P}= & \mathrm{D}_{x y} H^{*}(\boldsymbol{x}, \boldsymbol{m}(\boldsymbol{x})) \mathrm{D} \boldsymbol{m}(\boldsymbol{x}) \\
= & \left(\mathrm{D}_{\boldsymbol{x} y} H\left(\boldsymbol{x}, \boldsymbol{y}, u_{1}(\boldsymbol{x})\right)\right. \\
& \left.+\nabla u_{1}(\boldsymbol{x})\left(\nabla_{\boldsymbol{y}} H_{z}\left(\boldsymbol{x}, \boldsymbol{y}, u_{1}(\boldsymbol{x})\right)\right)^{\mathrm{T}}\right) \mathrm{D} \boldsymbol{m}(\boldsymbol{x}) .
\end{aligned}
$$

We define the matrix $\boldsymbol{C}=\mathrm{D}_{x y} H^{*}(\boldsymbol{x}, \boldsymbol{y})$, which we call the mixed Hessian matrix, and rewrite Eq. (42) as

$$
\boldsymbol{P}(\boldsymbol{x})=\boldsymbol{C}\left(\boldsymbol{x}, \boldsymbol{m}(\boldsymbol{x}), u_{1}(\boldsymbol{x})\right) \mathrm{D} \boldsymbol{m}(\boldsymbol{x}) .
$$

Assuming the mixed Hessian matrix $C$ is invertible, the mapping $\boldsymbol{m}(\boldsymbol{x})$ is given by the critical point of Eq. (40).

\section{NUMERICAL METHOD}

We first apply the iterative least-squares algorithm from [1] on Eq. (43) using $H=H_{1}$ in Eq. (28) to compute $u_{1}(\boldsymbol{x})=u(\boldsymbol{x})$ and $\boldsymbol{y}_{1}=\boldsymbol{m}_{1}(\boldsymbol{x})$. Subsequently, we substitute $u(\boldsymbol{x})$ and $\boldsymbol{m}_{1}(\boldsymbol{x})$ into $H_{2}$ in Eq. (34) and run the least-squares algorithm from [1] again on Eq. (43) using $H=H_{2}$ in Eq. (34) to compute $u_{1}(\boldsymbol{x})=v(\boldsymbol{x})$ and the composite mapping $\boldsymbol{y}_{2}=\boldsymbol{m}(\boldsymbol{x})=\left(\boldsymbol{m}_{2} \circ \boldsymbol{m}_{1}\right)(\boldsymbol{x})$. In the next section, we present a short summary of the least-squares procedure in [1] for a general $H$ and associated mapping written as $\boldsymbol{y}=\boldsymbol{m}(\boldsymbol{x})$.

\section{A. Generalized Least-Squares Algorithm}

In this section, we will give a short overview of the numerical algorithm to compute the mapping $\boldsymbol{m}$ in Eq. (33) (subscript dropped) or Eq. (36), and the surfaces $u_{1}=u$ or $u_{1}=v$, respectively, denoting $F=F_{1}$ or $F=F_{2}$, and using Eq. (40) with $H=H_{1}$ or $H=H_{2}$, respectively.

The computation of $\boldsymbol{m}$ is an iterative procedure; in every iteration, we minimize four functionals, displayed in Eq. (37). First, to solve Eq. (33a) or Eq. (36a), we use Eq. (43) with $H$ from Eq. (28) or Eq. (34), respectively, for the interior domain and minimize

$$
J_{I}[\boldsymbol{m}, \boldsymbol{P}]=\frac{1}{2} \int_{\mathcal{X}}\|\boldsymbol{C D} \boldsymbol{m}-\boldsymbol{P}\|^{2} \mathrm{~d} \boldsymbol{x},
$$

under the constraint $\operatorname{det}(\boldsymbol{P})=F(\cdot, \boldsymbol{m}) \operatorname{det}(\boldsymbol{C}(\cdot, \boldsymbol{m}))$. The norm used is the Frobenius norm. To impose the transport boundary condition in Eq. (33b) or Eq. (36b), we minimize the functional

$$
J_{B}[\boldsymbol{m}, \boldsymbol{b}]=\frac{1}{2} \oint_{\partial \mathcal{X}}|\boldsymbol{m}-\boldsymbol{b}|^{2} \mathrm{~d} s,
$$

where $|\cdot|$ denotes the $L_{2}$-norm, and $\boldsymbol{b}: \partial \mathcal{X} \rightarrow \partial \mathcal{Y}$. We minimize a weighted average of functionals $J_{I}$ and $J_{B}$ as

$$
J[\boldsymbol{m}, \boldsymbol{P}, \boldsymbol{b}]=\alpha J_{I}[\boldsymbol{m}, \boldsymbol{P}]+(1-\alpha) J_{B}[\boldsymbol{m}, \boldsymbol{b}],
$$

with $0<\alpha<1$ the weighting parameter. Last, to compute $u_{1}=$ $u_{1}(\boldsymbol{x})$ from $\boldsymbol{m}$, we use Eq. (39) and minimize the functional

$$
I\left[u_{1}, \boldsymbol{m}\right]=\frac{1}{2} \int_{\mathcal{X}}\left|\nabla_{\boldsymbol{x}} H\left(\boldsymbol{x}, \boldsymbol{m}, u_{1}\right)+H_{z}\left(\boldsymbol{x}, \boldsymbol{m}, u_{1}\right) \nabla u_{1}\right|^{2} \mathrm{~d} \boldsymbol{x} .
$$

We iterate starting from an initial guess $\boldsymbol{m}^{0}$ and mixed Hessian matrix $\boldsymbol{C}\left(\cdot, \boldsymbol{m}^{0}\right)$ :

$$
\begin{gathered}
\boldsymbol{b}^{n+1}=\operatorname{argmin}_{\boldsymbol{b} \in \mathcal{B}} J_{B}\left[\boldsymbol{m}^{n}, \boldsymbol{b}\right], \\
\boldsymbol{P}^{n+1}=\operatorname{argmin}_{\boldsymbol{P} \in \mathcal{P}\left(\boldsymbol{m}^{n}\right)} J_{I}\left[\boldsymbol{m}^{n}, \boldsymbol{P}\right], \\
\boldsymbol{m}^{n+1}=\operatorname{argmin}_{\boldsymbol{m} \in \mathcal{M}} J\left[\boldsymbol{m}, \boldsymbol{P}^{n+1}, \boldsymbol{b}^{n+1}\right], \\
u_{1}^{n+1}=\operatorname{argmin}_{u_{1} \in \mathcal{U}} I\left[u_{1}, \boldsymbol{m}^{n+1}\right],
\end{gathered}
$$

where the minimization steps are performed over the spaces

$$
\begin{gathered}
\mathcal{B}=\left\{\boldsymbol{b} \in C^{1}(\partial \mathcal{X})^{2} \mid \boldsymbol{b}(\boldsymbol{x}) \in \partial \mathcal{Y}\right\}, \\
\mathcal{P}(\boldsymbol{m})=\left\{\boldsymbol{P} \in C^{1}(\mathcal{X})^{2 \times 2} \mid \boldsymbol{P} \mathrm{SND},\right. \\
\operatorname{det}(\boldsymbol{P})=F(\cdot, \boldsymbol{m}) \operatorname{det}(\boldsymbol{C}(\cdot, \boldsymbol{m}))\}, \\
\mathcal{M}=C^{2}(\mathcal{X})^{2}, \\
\mathcal{U}=C^{2}(\mathcal{X}) .
\end{gathered}
$$

After each iteration, we compute the matrix $\boldsymbol{C}\left(\cdot, \boldsymbol{m}^{n+1}, u_{1}^{n+1}\right)$.

The minimization steps (45a)-(45d) are described in detail in $[1,5,6]$. The operations in Eqs. (45a) and (45b) are point-wise minimization steps. In contrast, the minimization step Eq. (45c) cannot be performed point-wise. Applying calculus of variations to $J$, we obtain a coupled elliptic boundary value problem for the two components $\boldsymbol{m}=\left(m^{1}, m^{2}\right)$, which can be written as

$$
\nabla \cdot\left(\boldsymbol{C}^{T} \boldsymbol{C D} \boldsymbol{m}\right)=\nabla \cdot\left(\boldsymbol{C}^{T} \boldsymbol{P}\right), \quad \boldsymbol{x} \in \mathcal{X},
$$

$(1-\alpha) \boldsymbol{m}+\alpha\left(\boldsymbol{C}^{T} \boldsymbol{C D} \boldsymbol{m}\right) \hat{\boldsymbol{n}}=(1-\alpha) \boldsymbol{b}+\alpha \boldsymbol{C P} \hat{\boldsymbol{n}}, \quad \boldsymbol{x} \in \partial \mathcal{X}$,

where $\hat{\boldsymbol{n}}$ is the normal outward to the surface. We discretize Eq. (47) using the finite volume method [6].

Applying calculus of variations to $I$, we obtain the Neumann problem

$$
\begin{gathered}
\nabla \cdot\left(H_{z} \nabla_{\boldsymbol{x}} H^{*}\right)=\frac{1}{2} \frac{\mathrm{d}}{\mathrm{d} z}\left|\nabla_{\boldsymbol{x}} H^{*}\right|^{2}, \quad \boldsymbol{x} \in \mathcal{X}, \\
H_{z} \nabla_{\boldsymbol{x}} H^{*} \cdot \hat{\boldsymbol{n}}=0, \quad \boldsymbol{x} \in \partial \mathcal{X} .
\end{gathered}
$$

Substituting $\boldsymbol{m}^{n+1}$ and the function $H\left(\boldsymbol{x}, \boldsymbol{m}^{n+1}, u_{1}\right)$ at iteration $n$, this is a Neumann problem for $u_{1}$ that has a corresponding finite volume matrix with incomplete rank. We calculate a unique solution by prescribing the average value of $u_{1}$ as a constraint. For the details, we refer to [1]. 


\section{NUMERICAL RESULTS}

In this section, we apply the generalized least-squares algorithm to two example problems. For both test cases, we choose $\alpha=0.2$ as a weighting parameter for functional $J$. The laptop used for the calculations has an Intel Core i7-7700HQ CPU $2.80 \mathrm{GHz}$ with $32 \mathrm{~GB}$ of RAM.

\section{A. Targets Corresponding to Known Surfaces}

To test the accuracy of the algorithm, we solve the generalized Monge-Ampère equations for a double freeform lens where we pre-compute the right-hand sides in Eqs. (33a) and (36a) corresponding to two known surfaces. We choose

$$
u(\boldsymbol{x})=1+|\boldsymbol{x}|^{2}, \quad v(\boldsymbol{x})=1,
$$

such that $u(\boldsymbol{x})$ represents an elliptic paraboloid. We use the implicit relation for the mapping in Eq. (39) to solve for the mappings $\boldsymbol{m}_{1}(\boldsymbol{x})$ and $\boldsymbol{m}(\boldsymbol{x})$ using Eq. (28) and Eq. (34), respectively. These are long expressions that are not included in this paper for brevity.

We consider a square source domain $\mathcal{X}=[-1,1]^{2}$. Using the mapping $\boldsymbol{m}_{1}(\boldsymbol{x})$, we compute the target domain $\mathcal{Y}_{1}$ and the corresponding target boundary. Using the mapping $\boldsymbol{m}(\boldsymbol{x})$, we compute the target domain $\mathcal{Y}_{2}$ and the corresponding target boundary. We use $H$ in Eq. (28) and compute the right-hand side $F_{1}\left(\boldsymbol{x}, \boldsymbol{m}_{1}(\boldsymbol{x}), u_{1}(\boldsymbol{x})\right)$ in Eq. (33a) using the exact $\boldsymbol{m}_{1}(\boldsymbol{x})$ and $u(\boldsymbol{x})$, which corresponds to any source intensity $\tilde{f}(\boldsymbol{x})$ and intermediate target intensity $\tilde{h}\left(\boldsymbol{y}_{1}\right)$, such that the last equality in Eq. (33a) holds. We use an initial mapping that maps the source to a bounding box around the target, and spherical initial surface as in [1], and run the algorithm to compute $u(\boldsymbol{x})$ and the intermediate mapping $\boldsymbol{m}_{1}(\boldsymbol{x})$. The results are shown in Fig. 4, in the left column.

After computing $\boldsymbol{m}_{1}(\boldsymbol{x})$ and $u(\boldsymbol{x})$, we run the algorithm again using $H$ in Eq. (34). We evaluate the right-hand side $F_{2}\left(\boldsymbol{x}, \boldsymbol{m}(\boldsymbol{x}), u_{1}(\boldsymbol{x})\right)$ in Eq. (36a) using the exact $\boldsymbol{m}(\boldsymbol{x})$ and $v(\boldsymbol{x})$, which corresponds to any source intensity $\tilde{f}(\boldsymbol{x})$ and target intensity $\tilde{g}\left(\boldsymbol{y}_{2}\right)$, such that the last equality in Eq. (36a) is satisfied. We use a G-convex initial mapping and constant initial $v$ as in [1], and run the algorithm to compute $v(\boldsymbol{x})$ and the composite mapping $\boldsymbol{m}(\boldsymbol{x})$. The results are shown in Fig. 4, in the right column.

We use refractive index $n=1.5$ and $\alpha=0.2$. Figures 4 (c) and $4(\mathrm{~d})$ show the difference in the mappings for the two components $m^{1}$ and $m^{2}$ with the exact solutions for several $N \times N$ grids with logarithmic least-squares fits. Figures $4(\mathrm{e})$ and $4(\mathrm{f})$ show the difference in the surfaces with the exact solutions. We observe a second-order convergence to the exact solution. The convergence of $J_{I}$ and $J_{B}$ is shown in Figs. $4(\mathrm{~g})$ and $4(\mathrm{~h})$. Note that functionals $J_{I}$ and $J_{B}$ reach a plateau at a certain iteration number, due to discretization and rounding errors. The first and second surfaces for $N=100$ are plotted in Fig. 4(i). The second surface deviates slightly from the exact solution, with the absolute difference displayed in Fig. 4(j). Details on the number of iterations and computation time of the algorithm are presented in Table 1, where we use the stopping criterion presented in [1].

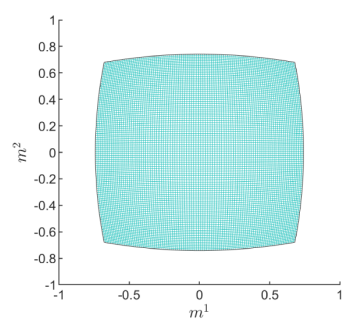

(a)
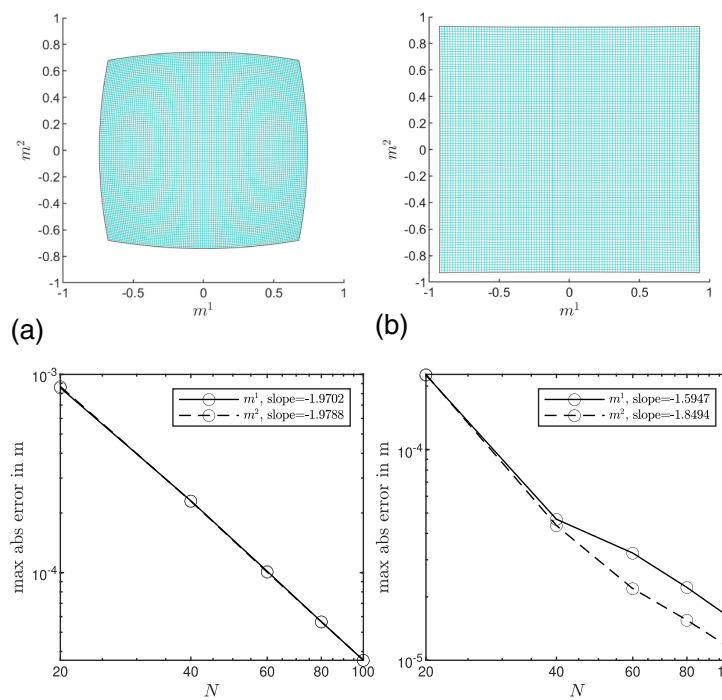

(c)

(b)

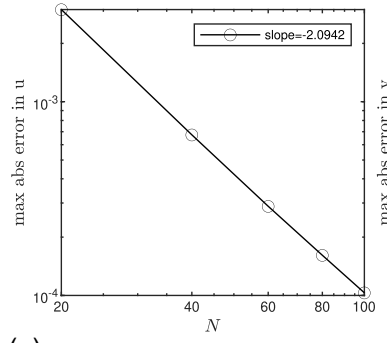

(e)

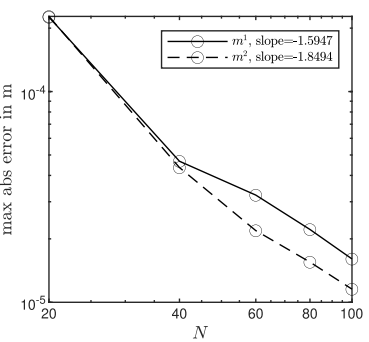

(d)

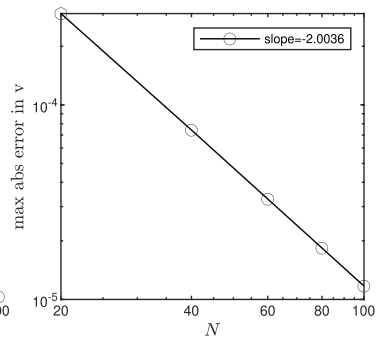

(f)

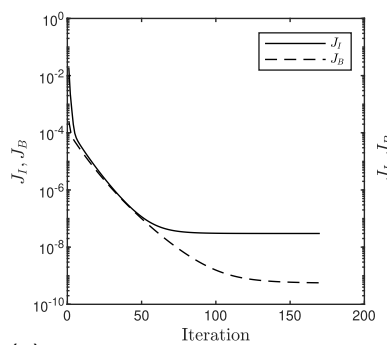

(g)

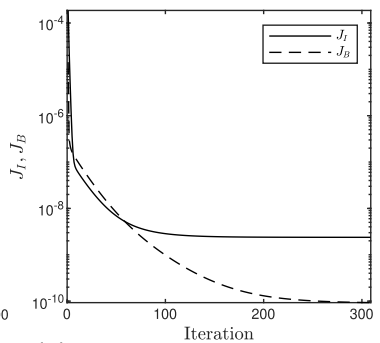

(h)

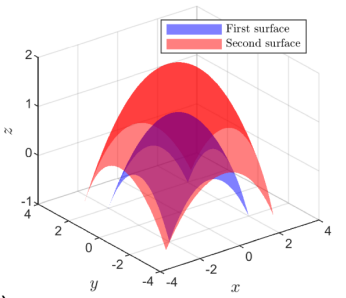

(i)

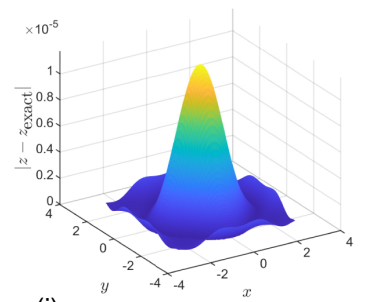

(j)

Fig. 4. "Double-freeform-lens" problem: we calculate solutions to $u$ and $v$ with parameter values $\alpha=0.2$ and $n=1.5$. (a) Mapping $m_{1}(x)$. (b) Mapping $m(x)$. (c) Maximum absolute error in $m_{1}(x)$. (d) Maximum absolute error in $m(x)$. (e) Maximum absolute error in $u(x)$. (f) Maximum absolute error in $v(x)$. (g) First surface error, $N=100$. (h) Second surface error, $N=100$. (i) First and second surfaces, $N=100$. (j) Absolute error second surface $N=100$.

The number of iterations required increases sublinearly with $N$. The computation time increases quadratically. Last, we see that $J_{I}$ and $J_{B}$ have approximately fourth-order convergence. 
Table 1. Number of Iterations, Total Computation Time (in Seconds), and Residuals in the Least-Squares Algorithm for the "Exact Lens" Problem

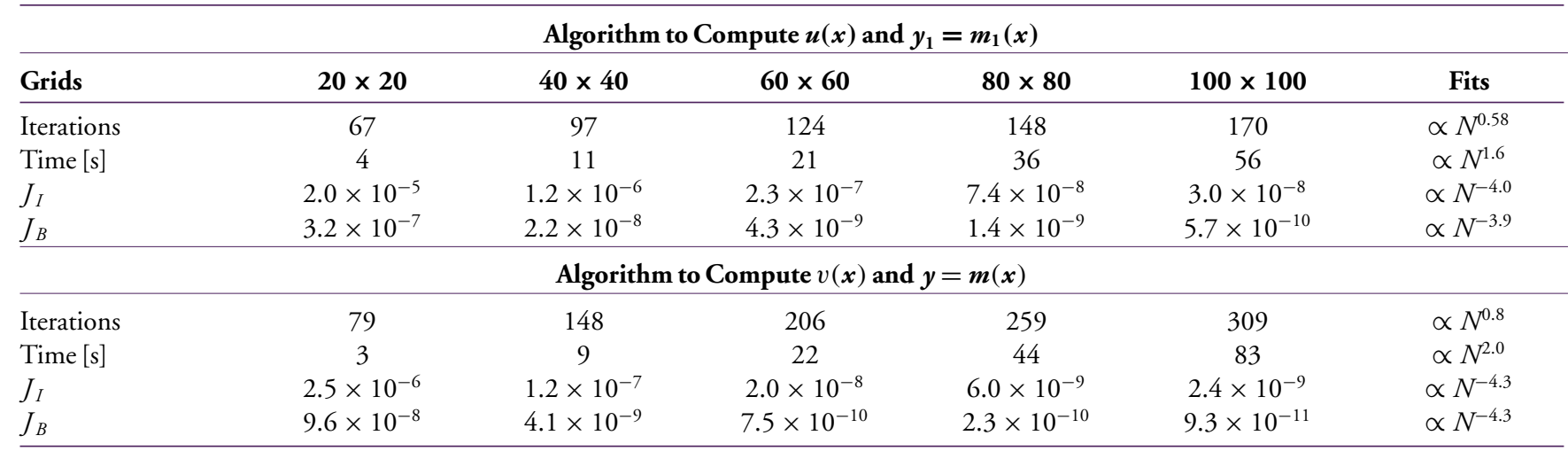

\section{B. Picture on a Projection Screen in the Far Field}

We consider a square source domain $\mathcal{X}=[-0.5,0.5]^{2}$ with a Lambertian source intensity $f(\phi, \theta)=\cos (\phi)$ inside the square, i.e., the emittance is proportional to the cosine of the zenith $\phi$ described in Section 2. Using Eq. (3a), we derive that $\tilde{f}(\boldsymbol{x})=\left(1-|\boldsymbol{x}|^{2}\right) /\left(1+|\boldsymbol{x}|^{2}\right)$ in stereographic coordinates.

The twice refracted rays are projected on a screen $P$ in the far field, parallel to the plane $z=0$. The required illuminance $L(\xi, \eta)\left[\mathrm{lm} / \mathrm{m}^{2}\right]$, with $(\xi, \eta)$ the Cartesian coordinates on the projection screen, is derived from the gray-scale values of a famous painting by Vermeer: "Girl with a Pearl Earring," 1665, Mauritshuis, The Hague [43]. The gray-scale picture has contrast ratio 22:1. The conversion from the colored image to gray-scale values creates black regions in the target distribution for which $\tilde{g}\left(\boldsymbol{y}_{2}\right)=0$. To avoid division by zero in the right-hand side of Eq. (36a), we increase values of $\tilde{g}\left(\boldsymbol{y}_{2}\right)$ that are below a threshold of $15 \%$ of its maximum value to this threshold. The updated contrast ratio is $6.7: 1$. The target distribution $\tilde{g}\left(\boldsymbol{y}_{2}\right)$ is a deformation of the illuminance $L(\xi, \eta)$; the conversion from $L(\xi, \eta)$ to $\tilde{g}\left(\boldsymbol{y}_{2}\right)$ is explained in detail in [3,30].

We discretize the source domain by a $250 \times 250$ grid and use the parameter values $\alpha=0.2$ and $n=1.5$.

To compute the first freeform surface, we need to choose an intermediate target intensity $\tilde{h}\left(\boldsymbol{y}_{1}\right)=h\left(\psi_{1}\left(\boldsymbol{y}_{1}\right), \chi_{1}\left(\boldsymbol{y}_{1}\right)\right)$. For this purpose, we consider the generating function

$$
G(\boldsymbol{x}, \boldsymbol{y}, z)=\boldsymbol{x} \cdot \boldsymbol{y}+z .
$$

Using this generating function, the generalized Monge-Ampère equation in Eq. (36a) reduces to the standard Monge-Ampère equation involving the determinant of the Hessian matrix of $u_{1}$, and the matrix $\boldsymbol{C}$ in Eq. (43) is the identity matrix (multiplied by a minus sign). We use the least-squares algorithm to compute a mapping $\boldsymbol{y}_{2}=\boldsymbol{m}^{*}(\boldsymbol{x})$ from $\mathcal{X}$ to $\mathcal{Y}_{2}$. Note that $u_{1}$ is not equal to $u$ or $v$ in this case, but a scalar potential of the mapping $\boldsymbol{m}^{*}$. The generating function in Eq. (50) describes optical systems involving a parallel incoming source beam and far-field target, for which the cost function in optimal transport theory is quadratic and can be written as $c(\boldsymbol{x}, \boldsymbol{y})=\boldsymbol{x} \cdot \boldsymbol{y}$ [44]. This simplifies the boundary value problems in Eqs. (47) and (48) and gives us an initial mapping $\boldsymbol{m}^{*}$ equivalent to the initial mapping used in most ray mapping methods. In fact, using this generating function, the numerical algorithm reduces to the first version presented in $[3,42]$. We consider the intermediate mapping

$$
\boldsymbol{m}_{1}^{*}=\beta \boldsymbol{x}+(1-\beta) \boldsymbol{m}^{*}, \quad 0 \leq \beta \leq 1
$$

as a weighted average of the identity mapping and the initial mapping $\boldsymbol{m}^{*}(\boldsymbol{x})$. Subsequently, we set the intermediate intensity $\tilde{h}$ as

$$
\tilde{h}\left(\boldsymbol{y}_{1}\right)=\frac{f(\boldsymbol{x})}{\operatorname{det}\left(\mathrm{D} \boldsymbol{m}_{1}^{*}(\boldsymbol{x})\right)} .
$$

We consider different values of $\beta$ ranging from zero to one. Choosing the intermediate target intensity in this way is akin to choosing Wasserstein barycentric averages with a quadratic cost function in [45]. The intermediate intensity $\tilde{h}$ can be interpreted as an intermediate target intensity that results from a collimated light source and a single optical surface.

Once we have computed $\tilde{h}\left(\boldsymbol{y}_{1}\right)$, we use $H_{1}$ in Eq. (28) and use the algorithm to solve the generalized Monge-Ampère equation Eq. (29) to compute the intermediate mapping $\boldsymbol{m}_{1}(\boldsymbol{x})$ and $u(\boldsymbol{x})$. Subsequently, we use $H_{2}$ in Eq. (34) and run the algorithm to solve Eq. (36) to compute the composite mapping $\boldsymbol{m}(\boldsymbol{x})$ and $v(\boldsymbol{x})$.

Figure 5 shows the results of the mappings and surfaces for various $\beta$. When $\beta=1$, the mapping $\boldsymbol{m}_{1}$ is simply the identity mapping, and $u$ is a spherical surface that does not alter the direction of the incoming rays. For smaller $\beta$, more detail of the Vermeer picture is incorporated in the first optical surface. Table 2 shows details on the number of iterations and computation times. The number of iterations and computation time to compute the first surface increase for smaller $\beta$, with a maximum computation time of $112 \mathrm{~s}$. To compute the second surface, the number of iterations and computation time are similar for all values of $\beta$, with a mean computation time of $55 \mathrm{~s}$.

In Fig. 6, the ray trace results of the lens are displayed, using a self-programmed ray tracing algorithm in MATLAB performed with $5 \times 10^{5}$ rays from quasi-random positions (quasi-Monte Carlo) on the source domain. We triangulate the surfaces $u$ and $v$, and for each ray, we calculate the intersection with the surfaces and corresponding normal vector to the faces of the triangles using the Möller-Trumbore ray-triangle intersection algorithm [46]. Subsequently, we compute the direction of the light rays $\hat{\imath}$ and $\hat{\boldsymbol{t}}$ using the vectorial law of refraction twice and determine 

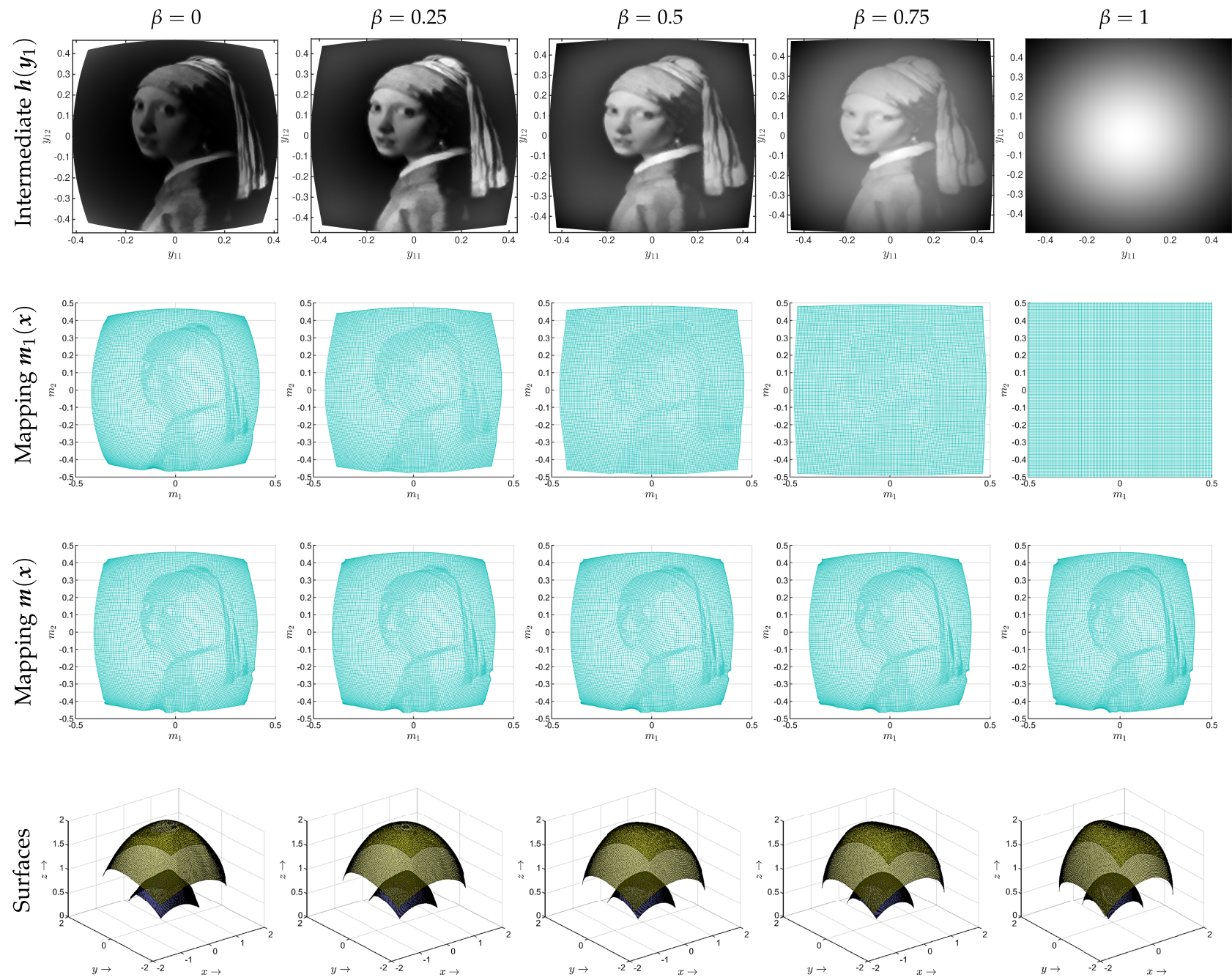

Fig. 5. "Vermeer-lens" problem: intermediate target intensities $\tilde{h}\left(\boldsymbol{y}_{1}\right)$, mappings $\boldsymbol{m}_{1}(\boldsymbol{x})$ and $\boldsymbol{m}(\boldsymbol{x})$, and optical surfaces $u(\boldsymbol{x})$ (bottom surface) and $v(\boldsymbol{x})$ (top surface) plotted in Euclidean space. Vermeer: Mauritshuis, The Hague [43].

Table 2. Number of Iterations, Total Computation Time (in Seconds), and Residuals in the Least-Squares Algorithm for the "Vermeer-Lens" Problem

\begin{tabular}{lccccc}
\hline \multicolumn{7}{c}{ Algorithm to Compute $\boldsymbol{u}(\boldsymbol{x})$ and $\boldsymbol{y}_{\mathbf{1}}=\boldsymbol{m}_{\mathbf{1}}(\boldsymbol{x})$} \\
\hline $\boldsymbol{\beta}$ & $\mathbf{0}$ & $\mathbf{0 . 2 5}$ & $\mathbf{0 . 5}$ & $\mathbf{0 . 7 5}$ & $\mathbf{1}$ \\
\hline Iterations & 87 & 63 & 51 & 47 & 10 \\
Time $[\mathrm{s}]$ & 112 & 91 & 64 & 61 & 13 \\
$J_{I}$ & $1.2 \times 10^{-3}$ & $6.2 \times 10^{-4}$ & $2.7 \times 10^{-4}$ & $6.5 \times 10^{-5}$ & $2.3 \times 10^{-26}$ \\
$J_{B}$ & $2.4 \times 10^{-5}$ & $1.1 \times 10^{-5}$ & $4.6 \times 10^{-6}$ & $9.7 \times 10^{-7}$ & $6.6 \times 10^{-27}$ \\
\hline \multicolumn{7}{c}{ Algorithm to Compute $v(\boldsymbol{x})$ and $\boldsymbol{y}=\boldsymbol{m}(\boldsymbol{x})$} \\
\hline Iterations & 49 & 48 & 46 & 45 & 44 \\
Time $[\mathrm{s}]$ & 56 & 61 & 51 & 55 & 51 \\
$J_{I}$ & $5.0 \times 10^{-2}$ & $4.8 \times 10^{-2}$ & $4.8 \times 10^{-2}$ & $4.7 \times 10^{-2}$ & $4.6 \times 10^{-2}$ \\
$J_{B}$ & $7.5 \times 10^{-5}$ & $9.0 \times 10^{-5}$ & $1.0 \times 10^{-4}$ & $1.2 \times 10^{-4}$ & $1.2 \times 10^{-4}$ \\
\hline
\end{tabular}

the corresponding bin on the target domain, divided into 200 $\times 200$ bins. The ray trace results match the picture of Vermeer for all $\beta$. None of the traced rays missed the first or the second surface. Note that in the generating function approach of this paper, we assume that each ray $\hat{\boldsymbol{s}}$ refracts at the optical surfaces and always reaches the target plane. Hence, we circumvent the occurrence of total internal reflection (TIR). The vectorial law of refraction (p. 140, [47]) gives

$$
\hat{\imath}=\frac{1}{n} \hat{\boldsymbol{s}}-\left(\frac{1}{n}\left(\hat{\boldsymbol{s}} \cdot \hat{\boldsymbol{n}}_{1}\right)+\sqrt{1-\frac{1}{n^{2}}\left(1-\left(\hat{\boldsymbol{s}} \cdot \hat{\boldsymbol{n}}_{1}\right)^{2}\right)}\right) \hat{\boldsymbol{n}}_{1},
$$



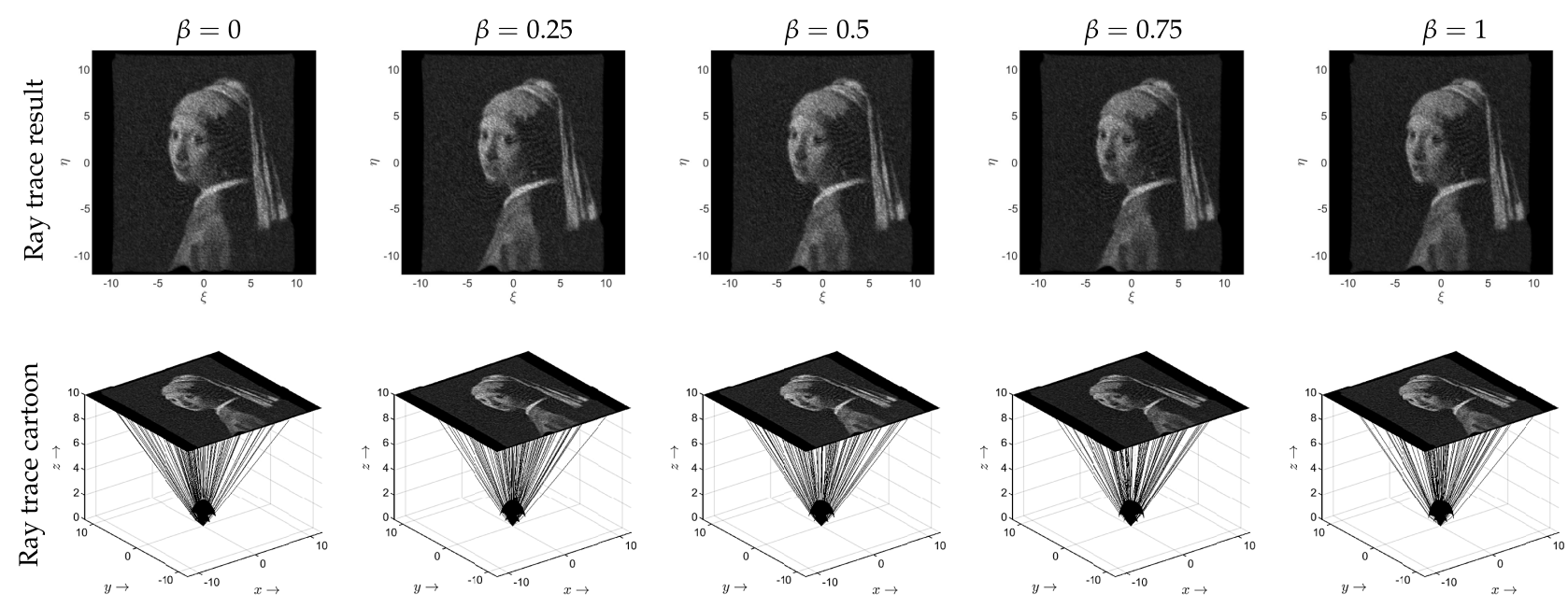

Fig. 6. "Vermeer-lens" problem: ray-traced images and optical surfaces, which are small relative to the distance the light rays travel to the projection screen in the far field, together with a subset of rays traced. Vermeer: Mauritshuis, The Hague [43].

$$
\hat{\boldsymbol{t}}=n \hat{\boldsymbol{\iota}}-\left(n\left(\hat{\boldsymbol{\imath}} \cdot \hat{\boldsymbol{n}}_{2}\right)+\sqrt{1-n^{2}\left(1-\left(\hat{\boldsymbol{\imath}} \cdot \hat{\boldsymbol{n}}_{2}\right)^{2}\right)}\right) \hat{\boldsymbol{n}}_{2}
$$

requiring that the terms under the square roots $1-1 / n^{2}(1-$ $\left.\left(\hat{\boldsymbol{s}} \cdot \hat{\boldsymbol{n}}_{1}\right)^{2}\right) \geq 0$ and $1-n^{2}\left(1-\left(\hat{\boldsymbol{\boldsymbol { l }}} \cdot \hat{\boldsymbol{n}}_{2}\right)^{2}\right) \geq 0$ for the first and second surfaces, respectively, where $\hat{\boldsymbol{n}}_{1}$ is the normal to the first surface and $\hat{\boldsymbol{n}}_{2}$ is the normal to the second surface. Both conditions are satisfied for all rays traced.

To verify the quality of the ray trace results, we take the difference between the target intensity on the projection screen (interpolated bilinearly onto the ray tracing grid) and the ray tracing irradiance. For all values of $\beta$, the RMS error is approximately 0.11 and the correlation 0.88 . Hence, for all values of $\beta$, the ray tracing irradiance closely matches the target intensity. This suggests that even for complicated and detailed target distributions, we can distribute the refractive power over both surfaces of the lens in any way we like.

\section{CONCLUSION}

In this paper, we presented a method to compute a double freeform lens that converts the light from a point source into a far-field target. Using Hamilton's characteristic functions, we derived the generating functions for both surfaces of the lens and showed that the optical mappings can be derived by considering G-convex and/or G-concave solutions for the locations of optical surfaces $u$ and $v$. Combining the optical mappings with energy conservation resulted in two generalized Monge-Ampère equations.

We used a least-squares approach to solve the generalized Monge-Ampère equations. First, we ran the algorithm using the generating function for $u$ to compute the optical mapping and surface $u$. Subsequently, we substituted the mapping and surface $u$ in the generating function for $v$ and re-ran the least-squares algorithm to compute the final mapping and surface $v$.

We tested the numerical algorithm on two example problems. For an exact solution, we checked the accuracy of the algorithm. We also tested the algorithm for a lens converting the light of a point source into a picture on a screen in the far field and verified the solution via ray tracing. We introduced a parameter to divide the required refraction over the first and second surfaces.

In future work, we will apply the method to a wider variety of real optical design problems, using various source and target distributions. For practical design examples, we would like to investigate the added flexibility of our numerical algorithm. By dividing the refractive power over two surfaces, we showed that we can compute multiple solutions. We would like to compare these multiple designs for qualities such as compactness and suitability for injection molding. In addition, we aim to explore another degree of freedom in our numerical procedure: computing other combinations of G-convex and G-concave surfaces for the first and second optical surfaces of the double freeform lens. Furthermore, we will consider extended light sources and take into account Fresnel reflections, scattering phenomena, and aberration theory.

Funding. Nederlandse Organisatie voor Wetenschappelijk Onderzoek (TTW Perspectief P15-36).

Disclosures. The authors declare no conflicts of interest.

\section{REFERENCES}

1. L. B. Romijn, J. H. M. ten Thije Boonkkamp, M. Anthonissen, and W. L. IJzerman, "An iterative least-squares method for generated Jacobian equations in freeform optical design," CASA Report 20 (2020), submitted to SIAM J. Sci. Comput.

2. N. S. Trudinger, "On the local theory of prescribed Jacobian equations," Discrete Contin. Dyn. Syst. 34, 1663-1681 (2012).

3. C. R. Prins, "Inverse methods for illumination optics," Ph.D. thesis (Eindhoven University of Technology, 2014).

4. N. K. Yadav, J. H. M. ten Thije Boonkkamp, and W. L. IJzerman, "A Monge-Ampère problem with non-quadratic cost function to compute freeform lens surfaces," J. Sci. Comput. 80, 475-499 (2019).

5. L. B. Romijn, J. H. ten Thije Boonkkamp, and W. L. IJzerman, "Inverse reflector design for a point source and far-field target," J. Comput. Phys. 408, 109283 (2020).

6. L. B. Romijn, J. H. M. ten Thije Boonkkamp, and W. L. IJzerman, "Freeform lens design for a point source and far-field target," J. Opt. Soc. Am. A 36, 1926-1939 (2019). 
7. C. Bösel and H. Gross, "Single freeform surface design for prescribed input wavefront and target irradiance," J. Opt. Soc. Am. A 34, 14901499 (2017).

8. K. Brix, Y. Hafizogullari, and A. Platen, "Designing illumination lenses and mirrors by the numerical solution of Monge-Ampère equations," J. Opt. Soc. Am. A 32, 2227-2236 (2015).

9. K. Brix, Y. Hafizogullari, and A. Platen, "Solving the Monge-Ampère equations for the inverse reflector problem," Math. Models Methods Appl. Sci. 25, 803-837 (2015).

10. R. Wu, L. Xu, P. Liu, Y. Zhang, Z. Zheng, H. Li, and X. Liu, "Freeform illumination design: a nonlinear boundary problem for the elliptic Monge-Ampère equation," Opt. Lett. 38, 229-231 (2013).

11. D. A. Bykov, L. L. Doskolovich, A. A. Mingazov, E. A. Bezus, and N. L. Kazanskiy, "Linear assignment problem in the design of freeform refractive optical elements generating prescribed irradiance distributions," Opt. Express 26, 27812-27825 (2018).

12. D. A. Bykov, L. L. Doskolovich, and E. A. Bezus, "Multiscale approach and linear assignment problem in designing mirrors generating far-field irradiance distributions," Opt. Lett. 45, 3549-3552 (2020).

13. L. L. Doskolovich, D. A. Bykov, E. S. Andreev, E. A. Bezus, and V. Oliker, "Designing double freeform surfaces for collimated beam shaping with optimal mass transportation and linear assignment problems," Opt. Express 26, 24602-24613 (2018).

14. L. L. Doskolovich, D. A. Bykov, A. A. Mingazov, and E. A. Bezus, "Optimal mass transportation and linear assignment problems in the design of freeform refractive optical elements generating far-field irradiance distributions," Opt. Express 27, 13083-13097 (2019).

15. T. Glimm and V. I. Oliker, "Optical design of single reflector systems and the Monge-Kantorovich mass transfer problem," J. Math. Sci. 117, 4096-4108 (2003).

16. C. E. Gutiérrez, "Refraction problems in geometric optics," in Fully Nonlinear PDEs in Real and Complex Geometry and Optics (Springer, 2014), pp. 95-150.

17. C. E. Gutiérrez and Q. Huang, "The refractor problem in reshaping light beams," Arch. Ration. Mech. Anal. 193, 423-443 (2009).

18. A. M. Oberman and Y. Ruan, "An efficient linear programming method for optimal transportation," arXiv:1509.03668 (2015).

19. V. Oliker, L. L. Doskolovich, and D. A. Bykov, "Beam shaping with a plano-freeform lens pair," Opt. Express 26, 19406-19419 (2018).

20. V. Oliker, J. Rubinstein, and G. Wolansky, "Supporting quadric method in optical design of freeform lenses for illumination control of a collimated light," Adv. Appl. Math. 62, 160-183 (2015).

21. X.-J. Wang, "On the design of a reflector antenna II," Calc. Var. Partial Differential Equations 20, 329-341 (2004).

22. N. K. Yadav, L. B. Romijn, J. H. M. ten Thije Boonkkamp, and W. L. IJzerman, "A least-squares method for the design of two-reflector optical systems," J. Phys. 1, 034001 (2019).

23. C. Canavesi, W. J. Cassarly, and J. P. Rolland, "Target flux estimation by calculating intersections between neighboring conic reflector patches," Opt. Lett. 38, 5012-5015 (2013).

24. K. Desnijder, P. Hanselaer, and Y. Meuret, "Ray mapping method for off-axis and non-paraxial freeform illumination lens design," Opt. Lett. 44, 771-774 (2019).

25. Z. Feng, B. D. Froese, and R. Liang, "Freeform illumination optics construction following an optimal transport map," Appl. Opt. 55, 4301-4306 (2016)

26. F. R. Fournier, W. J. Cassarly, and J. P. Rolland, "Fast freeform reflector generation using source-target maps," Opt. Express 18, 5295-5304 (2010).
27. D. Ma, Z. Feng, and R. Liang, "Freeform illumination lens design using composite ray mapping," Appl. Opt. 54, 498-503 (2015).

28. D. Michaelis, P. Schreiber, and A. Bräuer, "Cartesian oval representation of freeform optics in illumination systems," Opt. Lett. 36, 918920 (2011)

29. J. C. Miñano, "Application of the conservation of Etendue theorem for 2-d subdomains of the phase space in nonimaging concentrators," Appl. Opt. 23, 2021-2025 (1984).

30. M. Born and E. Wolf, Principles of Optics, 7th ed. (Cambridge University, 1999).

31. Y. Zhang, R. Wu, P. Liu, Z. Zheng, H. Li, and X. Liu, "Double freeform surfaces design for laser beam shaping with Monge-Ampère equation method," Opt. Commun. 331, 297-305 (2014).

32. V. Oliker, "Optical design of freeform two-mirror beam-shaping systems," J. Opt. Soc. Am. A 24, 3741-3752 (2007).

33. M. A. Moiseev, E. V. Byzov, S. V. Kravchenko, and L. L. Doskolovich, "Design of LED refractive optics with predetermined balance of ray deflection angles between inner and outer surfaces," Opt. Express 23, A1140-A1148 (2015).

34. Z. Feng, L. Huang, G. Jin, and M. Gong, "Designing double freeform optical surfaces for controlling both irradiance and wavefront," Opt. Express 21, 28693-28701 (2013).

35. Z. Feng, B. D. Froese, C.-Y. Huang, D. Ma, and R. Liang, "Creating unconventional geometric beams with large depth of field using double freeform-surface optics," Appl. Opt. 54, 6277-6281 (2015).

36. P. Gimenez-Benitez, J. C. Miñano, J. Blen, R. M. Arroyo, J. Chaves, O. Dross, M. Hernández, and W. Falicoff, "Simultaneous multiple surface optical design method in three dimensions," Opt. Eng. 43, 14891503 (2004).

37. S. Wei, Z. Zhu, Z. Fan, Y. Yan, and D. Ma, "Double freeform surfaces design for beam shaping with non-planar wavefront using an integrable ray mapping method," Opt. Express 27, 26757-26771 (2019).

38. A. Bruneton, A. Bäuerle, R. Wester, J. Stollenwerk, and P. Loosen, "High resolution irradiance tailoring using multiple freeform surfaces," Opt. Express 21, 10563-10571 (2013).

39. J. C. Miñano and J. C. Gonzalez, "New method of design of nonimaging concentrators," Appl. Opt. 31, 3051-3060 (1992).

40. H. Ries and J. Muschaweck, "Tailored freeform optical surfaces," J. Opt. Soc. Am. A 19, 590-595 (2002).

41. R. K. Luneburg, Mathematical Theory of Optics (University of California, 1964).

42. C. R. Prins, R. Beltman, J. H. M. ten Thije Boonkkamp, W. L. IJzerman, and T. W. Tukker, "A least-squares method for optimal transport using the Monge-Ampère equation," SIAM J. Sci. Comput. 37, B937-B961 (2015).

43. Mauritshuis, The Hague, "Girl with a pearl earring" (c. 1665) [Online; accessed December 4, 2020].

44. J. ten Thije Boonkkamp, L. Romijn, and W. IJzerman, "Generalized Monge-Ampère equations for illumination freeform design," Proc. SPIE 11185, 1118504 (2019).

45. N. Bonneel, G. Peyré, and M. Cuturi, "Wasserstein barycentric coordinates: histogram regression using optimal transport," ACM Trans. Graph. 35, 71 (2016).

46. T. Möller and B. Trumbore, "Fast, minimum storage ray-triangle intersection," J. Graph. Tools 2, 21-28 (1997).

47. A. S. Glassner, An Introduction to Ray Tracing (Elsevier, 1989). 